\title{
PARALLEL COMPUTATIONAL OPTIMIZATION IN OPERATIONS RESEARCH: A NEW INTEGRATIVE FRAMEWORK, LITERATURE REVIEW AND RESEARCH DIRECTIONS
}

submitted to European Journal of Operational Research

\author{
Guido Schryen* \\ Department of Management Information Systems \\ Paderborn University, Germany \\ guido.schryen@upb.de \\ www. misor.org
}

October 9, 2019

\begin{abstract}
Solving optimization problems with parallel algorithms has a long tradition in OR. Its future relevance for solving hard optimization problems in many fields, including finance, logistics, production and design, is leveraged through the increasing availability of powerful computing capabilities. Acknowledging the existence of several literature reviews on parallel optimization, we did not find reviews that cover the most recent literature on the parallelization of both exact and (meta)heuristic methods. However, in the past decade substantial advancements in parallel computing capabilities have been achieved and used by OR scholars so that an overview of modern parallel optimization in OR that accounts for these advancements is beneficial. Another issue from previous reviews results from their adoption of different foci so that concepts used to describe and structure prior literature differ. This heterogeneity is accompanied by a lack of unifying frameworks for parallel optimization across methodologies, application fields and problems, and it has finally led to an overall fragmented picture of what has been achieved and still needs to be done in parallel optimization in OR. This review addresses the aforementioned issues with three contributions: First, we suggest a new integrative framework of parallel computational optimization across optimization problems, algorithms and application domains. The framework integrates the perspectives of algorithmic design and computational implementation of parallel optimization. Second, we apply the framework to synthesize prior research on parallel optimization in OR, focusing on computational studies published in the period 2008-2017. Finally, we suggest research directions for parallel optimization in OR.
\end{abstract}

Keywords computing science $\cdot$ parallel optimization $\cdot$ computational optimization $\cdot$ literature review

\section{Introduction}

Parallel optimization has received attention in the operations research (OR) field already for decades. Drawing on algorithmic and computational parallelism in OR is appealing as real-life optimization problems in a broad range of application domains are usually NP-hard and even the implementation of (meta)heuristic optimization procedures may require substantial computing resources. It has been argued that parallelism is crucial to make at least some problem instances tractable in practice and to keep computation times at reasonable levels [Talbi, 2009, Crainic et al. 2006]. $]^{2}$ However, unsurprisingly, the application of parallel optimization has been hesitant because i) parallelizing algorithms

\footnotetext{
*I am grateful for the support provided by Abdullah Burak, Philip Empl, Constanze Hilmer, Gerhard Rauchecker, Richard Schuster, Henning Siemes, and Melih Yilmaz, who supported me substantially in searching and coding research articles.

${ }^{2}$ Impressive computational results of applying parallelization to the traveling salesman problem (TSP) are reported by Crainic et al. [2006, p.2].
} 
is challenging in general from both the algorithmic and the computational perspective, and ii) a viable alternative to parallelizing algorithms has been the exploitation of ongoing increases of clock speed of single CPUs of modern microprocessors. But this growth process reached a limit already several years ago due to heat dissipation and energy consumption issues [Diaz et al. 2012]. This development makes parallelization efforts (not only in optimization) much more important than it was in earlier times.

Fortunately, the need for parallelization has been acknowledged and accompanied by an increased availability of parallel computing resources. This availability is rooted in two phenomena: a) the rapid development of parallel hardware architectures and infrastructures, including multi-core CPUs and GPUs, local high-speed networks and massive data storage, and of libraries and software frameworks for parallel programming [Talbi |2009. Crainic et al. 2006, Brodtkorb et al. [2013]; b) the increased availability of parallel computing resources as commodity good to researchers, who have (free or low-priced) access to multi-core laptops and workstations, and even to high-performance clusters offered by universities and public cloud providers.

The benefits of exploiting parallel processing for optimization algorithms are multi-faceted. Searching the solution space can be speeded up for both exact and (meta)heuristic algorithms so that the optimal solution or a given aspiration level of solution quality, respectively, can be achieved quicker. Implementations can also benefit from improved quality of the obtained solutions, improved robustness, and solvability of large-scale problems [Talbi, 2009, p. 460f].

We found many published reviews on parallel optimization for particular problems, methodologies, applications, research disciplines, and technologies. Reviews of parallelization for particular optimization problems were provided for one-dimensional integer knapsack problems [Gerasch and Wang, 1994], vehicle routing problems (VRPs) [Crainic, 2008], non-linear optimization [Lootsma and Ragsdell [1988], mixed integer programming [Nwana and Mitra| 2000] and multiobjective optimization [Nebro et al..|2005]. Most of the reviews that we found focus on parallel optimization regarding particular methodologies. While branch-and-bound algorithms have been reviewed by Gendron and Crainic [1994], the majority of methodological literature reviews have focused on metaheuristics: reviews have addressed tabu search (TS) [Crainic et al., 2005], simulated annealing (SA)[Aydin and Yigit, 2005], variable neighborhood search (VNS) [Pérez et al., 2005], Greedy Randomized Adaptive Search Procedures (GRASPs) [Resende and Ribeiro, 2005], swarm intelligence algorithms [Tan and Ding, 2016], particle swarm optimization algorithms [Zhang et al., 2015], and different types of evolutionary algorithms, including genetic algorithms (GAs) [Adamidis 1994. Luque et al. 2005. Cantú-Paz, 1998, Alba and Troya, 1999, Adamidis, 1994, Knysh and Kureichik, 2010], ant colony optimization algorithms [Pedemonte et al. |2011, Janson et al., 2005], scatter search [López et al.||2005] and evolutionary strategies |Rudolph, 2005|. Several reviews have covered sets of metaheuristics [Cung et al.|, 2002, Alba et al., 2005, Crainic and Hail |2005, Pardalos et al., 1995, Crainic and Toulouse, 2003, 2010, Crainic et al., 2014, Crainic, 2018, 2019, Alba et al., 2013] and hybrid metaheuristics [Cotta et al., 2005, Luna et al., 2005]. Application- and discipline-oriented reviews of parallel optimization have been provided for routing problems in logistics [Schulz et al., 2013] and for parallel metaheuristics in the fields of telecommunications and bioinformatics [Nesmachnow et al., 2005. Trelles and Rodriguez, 2005. Martins and Ribeiro, 2006]. Reviews that focus on particular parallelization technologies (in particular, General Purpose Computation on Graphics Processing Unit (GPGPU)) have been proposed by Boyer and El Baz] [2013], Tan and Ding [2016] and Schulz et al. [2013].

We acknowledge the excellent work provided in these reviews, from which our review has benefited substantially. At the same time, we see several arguments that call for a new literature review. First, we did not find reviews that cover the most recent literature on the parallelization of both exact and (meta)heuristic methods published in the decade 2008-2017. During this time, substantial advancements in parallel computing capabilities and infrastructures have been achieved and used by many OR scholars so that an overview of modern parallel optimization in OR that accounts for these advancements when synthesizing and classifying the literature is beneficial. Second, based on different foci adopted in previous literature reviews, the concepts used to describe and structure prior literature differ. This heterogeneity is accompanied by a lack of unifying frameworks for describing parallel optimization across methodologies, application fields, and problems. This has led finally to an overall fragmented picture of what has been achieved and what still needs to be done in parallel optimization in OR. As a side effect, the heterogeneity with which parallelization studies in OR have been described in terms of algorithmic parallelization, computational parallelization and performance of parallelization is high, which is beneficial from a diversity perspective but also raises problems: First, it remains unclear for authors what should be reported in an OR study that draws on parallel optimization; second, our own experience based on screening and reading several hundreds of articles is that the heterogeneity makes it often time-consuming and in some case even impossible for readers to identify the aforementioned parallelization characteristics of a study, to classify the study accordingly and to compare studies with each other.

Accounting for the aforementioned challenges, we provide three contributions in this literature review. First and to our best knowledge, we suggest the first universally applicable framework for parallel optimization in OR, which can be used by researchers to systematically describe their parallelization studies and position these in the landscape of 
parallel optimization without requirements on the application domain touched, the problem addressed, the methodology parallelized or the technology applied. In particular, the suggested framework integrates both algorithmic design and computational implementation issues of parallel optimization, which are usually being addressed separately in the literature. Second, we apply the integrative framework to synthesize prior research on parallel optimization in the field of OR published in the decade 2008-2017, focusing on those studies which include computational experiments. Finally, we suggest research directions, including recommendations, for prospective studies on parallel optimization in OR.

We structure our review as follows: In Section 2, we develop a framework for computational studies on parallel optimization. In Section 3, we define the scope and literature selection process of our review, before we review the literature in Section 4 based on the suggested framework. We provide research directions for future research in Section 5 before we conclude our review in Section 6

\section{Parallelization Framework}

Computational studies on parallel optimization usually report on four perspectives of parallelization [Gendron and Crainic, 1994, Alba and Luque, 2005, Crainic and Hail, 2005, Talbi, 2009, Pedemonte et al., 2011, Crainic, 2018, 2019]: object of parallelization, algorithmic parallelization, computational parallelization and performance of parallelization. While our review of the literature revealed that most studies make either implicitly or explicitly use of the aforementioned perspectives, we also observed a high level of heterogeneity in terms of terminology, taxonomies of parallel algorithmic design, granularity of information on parallel implementation, and performance metrics used to report computational results. As a consequence, with an increasing body of computational studies, it has become challenging to gain an overview of computational achievements, to compare studies in terms of their achievements, to develop consistent taxonomies for computational studies, and to identify white spots that need further research.

In order to mitigate the aforementioned problems in the field of parallel optimization, we suggest a new descriptive framework of computational parallel optimization studies (see Figure 11). The scope of the applicability of the proposed framework in the area of parallel optimization is wide with regard to two dimensions: First, it does not make any assumptions about the addressed application domain, the optimization problem to solve, the parallelized methodology or the applied technology. We denote this broad applicability as horizontal integration, referring to the horizontal layers in Figure 1. Second, it integrates the aforementioned perspectives (layers) and is based on well-established principles in the literature on algorithmic and computational parallelization. Similarly, we refer to this broad applicability as vertical integration, which brings together the - usually separately applied - perspectives on parallel optimization found in the disciplines of OR and computer science. In this context, our framework adopts an integrated view on parallel optimization. 


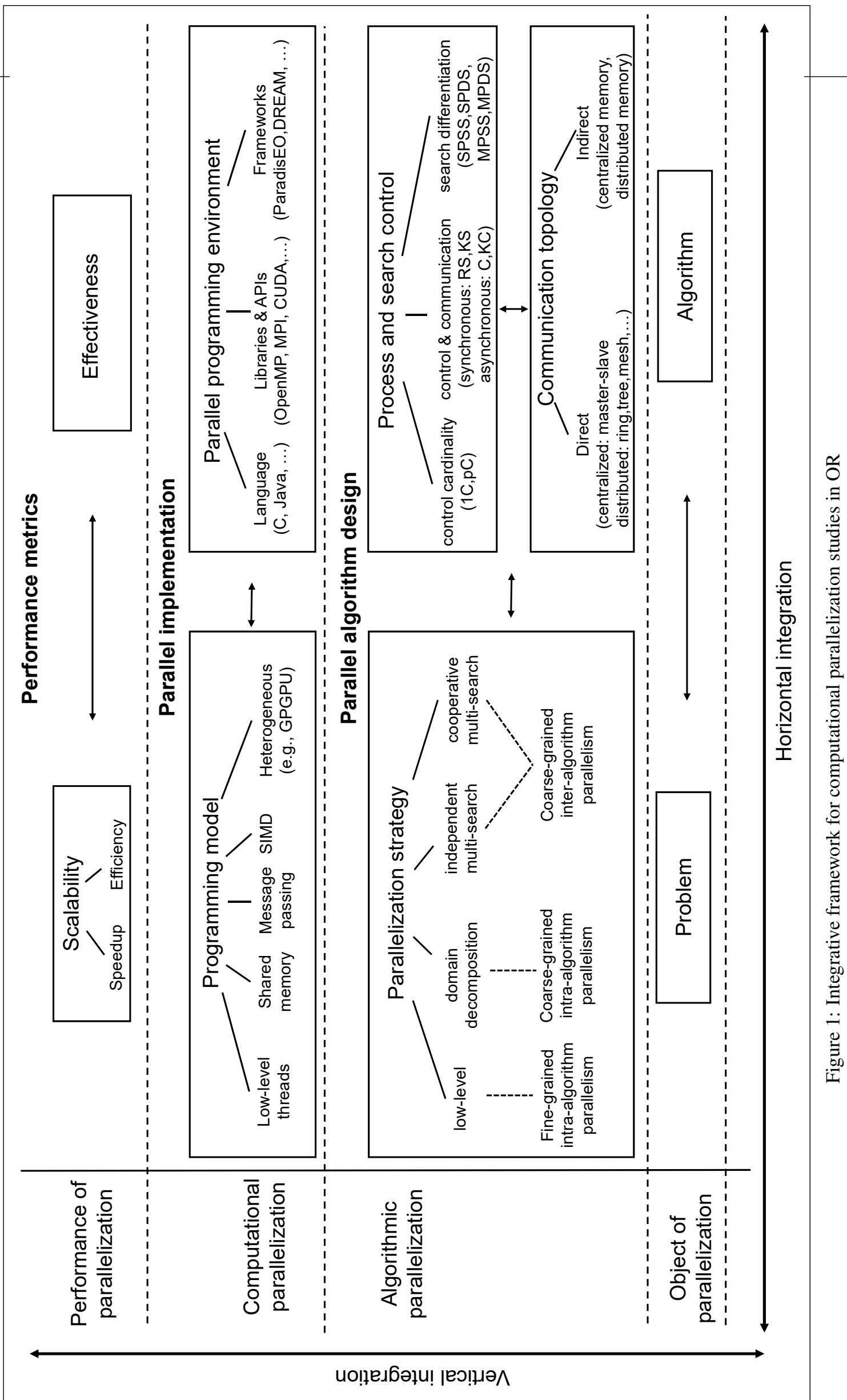




\subsection{Object of parallelization}

The object of parallelization comprises the OR problem to be solved (e.g., TSP, VRP, JSSP) and the algorithm to be applied (e.g., b\&b, GA, SA, TS), which effect each other. Problem types and algorithm types are both described in detail in Section 4.2

\subsection{Algorithmic parallelization}

The algorithmic parallelization refers to the methodological perspective on how parallelism is applied to solve an optimization problem by decomposition. As suggested for metaheuristics [Crainic 2019], we detail this perspective by distinguishing various types of parallelization strategy, process and search control, and communication topology (see Figure 17). Parallelization strategies have been defined according to the source of parallelism [Cung et al., 2002. Crainic and Toulouse, 2003, Crainic and Hail, 2005, Crainic and Toulouse, 2010, Crainic, 2019]. Four types are distinguished: (1) Functional parallelism applies when decomposition occurs at the algorithm level by, for example, evaluating neighbor solutions or computing the fitness of a solution in parallel. This parallelization strategy does not alter the algorithmic logic, the search space or the behavior of the sequential version, and it is thus also referred to as low-level. As parallelism occurs at a low level inside a single algorithm, we coin the term fine-grained intra-algorithm parallelism. Since the overall search follows only a single search path, this type of parallelism has also been denoted as single-walk parallelization, in contrast to the following strategies, where the overall search follows multiple trajectories and are referred to as multiple-walk parallelization strategies [Cung et al., 2002]. (2) Domain decomposition refers to the approach of separating and exploring the search space explicitly yielding a number of smaller and easier to solve subproblems to be addressed simultaneously by applying the same sequential algorithm. The partial solutions are finally used to reconstruct an entire solution of the original problem. The separation of the search space may be obtained, for example, by discarding or fixing variables and constraints. This separation may result in a partition (disjoint subsets) or a coverage (subsets may overlap) of the overall search space. In contrast to the low-level strategy, where parallelism occurs at a local and predefined part of the algorithm, domain decomposition involves concurrent explorations of subspaces using the same algorithm. Thus, we introduce the term coarse-grained intra-algorithm parallelism. (3) Separating the search space can also be performed implicitly through concurrent explorations of the search space by different or differently parameterized methods. When the concurrent execution of methods does not involve any exchange of information prior to identifying the best overall solution at the final synchronization step, the parallelization strategy is referred to as independent multi-search, which can be perceived as coarse-grained inter-algorithm parallelism. (4) When the concurrent execution of methods and their explorations of subspaces involves the exchange of information through cooperation mechanisms while the search process is in progress, cooperative multi-search occurs. The sharing of information may even be accompanied with the creation of new information out of exchanged data. As the interactions of the cooperative search algorithms specify the global search behavior, a new metaheuristic in its own right emerges [Crainic and Toulouse, 2008]. While cooperation yields in many cases a collective output with better solutions than a parallel independent search [Crainic |2019], exchanges should not be too frequent to avoid communication overheads and premature "convergence" to local optima [Toulouse et al., 2000, 2004]. As in the case of independent multi-search, also cooperative multi-search can be seen as coarse-grained inter-algorithm parallelism. Finally, it should be noticed that parallelization strategies are not mutually incompatible and may be combined into comprehensive algorithmic designs [Crainic et al. 2006, Crainic, 2019]. For example, low-level and decomposition parallelism have been jointly applied to branch-and-bound [Adel et al..|2016] and dynamic programming [Vu and Derbel, 2016], [Maleki et al., 2016], and low-level parallelism and cooperative multi-search have been applied to a hybrid metaheuristic [Munawar et al. 2009] which uses a genetic algorithm and hill climbing.

While the aforementioned parallelization strategies have been formulated for the class of metaheuristics, the strategydefining principles are of general nature of parallelizing optimization algorithms so that the scope of applicability of the parallelization strategies can be straightforward extended to other algorithm classes, including exact methods and (problem-specific) heuristics. For example, Gendron and Crainic [1994] have defined three types of parallelism for branch-and-bound: their type 1 parallelism refers to parallelism when performing operations on generated subproblems, such as executing the bounding operation in parallel for each subproblem. This type can be perceived as low-level parallelism. Parallelism of type 2 consists of building the branch-and-bound tree in parallel by performing operations on several subproblems concurrently. This type of parallelism involves an explicit separation of the search space and can, thus, be perceived as domain decomposition. Finally, the case of type 3 parallelism implies that several branch-and-bound trees are built in parallel, with the trees being characterized by different operations (branching, bounding, testing for elimination, or selection). This parallelism includes the option to use the information generated during the construction of a tree for the construction of another one. When such information is exchanged, type 3 parallelism can be perceived as cooperative multi-search, otherwise it corresponds to independent multi-search. The straightforward matching of parallelization strategies for metaheuristics with types of parallelism defined for an exact 
method supports our previous argument that the four parallelization strategies can be applied to the general "universe" of optimization algorithms.

Process and search control refers to how the global problems-solving process is controlled, how concurrent processes communicate with each other, and how diverse the overall search process is. We adopt the three dimensions suggested by Crainic and Hail [2005]: Search control cardinality determines whether the global search is controlled by a single process (1-control, 1C)) or by several processes (p-control, pC) which may collaborate or not. Search control and communications refers to how information is exchanged between processes and distinguishes between synchronous and asynchronous communication. In the former case, all concerned processes have to stop and engage in some form of communication and information exchange at specified moments (e.g., number of iterations) exogenously determined. In the latter case, processes are in charge of their own search as well as of establishing communications with other processes, and the global search terminates once each individual search stops. Both synchronous and asynchronous communication can be further qualified with regard to whether additional knowledge is derived from communication, leading to four categories of control and communication: rigid (RS) and knowledge synchronization (KS) in the synchronous case, and collegial (C) and knowledge collegial (KC) in the asynchronous case. Finally, the diversity of search may vary according to whether concurrently executed methods start from the same or different solutions, and to whether their search follows the same or different logic 3 , the diversity of search is also referred to as search differentiation. From these two dimensions the following four classes can be derived: 1. same initial point/population, same search strategy (SPSS); 2.same initial point/population, different search strategies (SPDS); 3. multiple initial points/populations, same search strategies (MPSS); 4. multiple initial points/populations, different search strategies $(M P D S)$. While the term "point" relates to single-solution methods, the notion "population" is used for population-based ones, such as genetic algorithms or ant colony optimizations. As in the case of parallelization strategies described above, the three dimensions of process and search control have been suggested for the classification of metaheuristics [Crainic and Hail, 2005, Crainic, 2018, 2019] but can be extended straightforward to other classes of optimization algorithms.

When concurrent processes exchange information, they may communicate with each other in a direct or indirect way. Direct communication involves message-based communication along some communication topology, such as a tree, ring, or fully connected mesh [Talbi, 2009, Crainic, 2019]. This communication topology needs to be projected on a physical interconnection topology as part of the implementation design. In contrast, indirect communication involves the use of a centralized or distributed memory, which are used as shared data resources of concurrent processes [Crainic, 2019].

The three perspectives of parallel algorithm design, namely parallelization strategy, process and search control, and communication topology, are linked together [Crainic, 2018, 2019]. Low-level parallelization is generally targeted in $1 \mathrm{C} / \mathrm{RS} / \mathrm{SPSS}$ designs, with the 1C (control cardinality) being implemented with a master-slave approach. Examples are the neighborhood evaluation of a local search heuristic, and the application of operators and the determination of fitness values in a GA. Domain decomposition is often implemented using a master-slave 1C/RS scheme with MPSS or MPDS search differentiation but can also be performed in a $\mathrm{pC}$, collegial decision making framework with MPSS or MPDS search differentiation. Independent multi-search is inherently a pC parallelization strategy, which follows from the same or different starting point(s)/population(s) with or without different search strategies (i.e., SPDS, MPSS or MPDS search differentiation). As the concurrently executed search processes do not exchange information prior to the final step, they follow the RS control and communication paradigm. Finally, cooperative multi-search is also a pC parallelization strategy, which may start from possibly different starting points/populations and may follow different search strategies (i.e., SPDS, MPSS or MPDS search differentiation). In contrast to independent multi-search, information is exchanged between processes during the search. This exchange of information can vary in different ways, which results in a large diversity of cooperation mechanisms. First, different types of information may be exchanged, including "good" solutions and context information. Second, cooperating processes may exchange information directly by sending messages to each other based on a given communication topology, or indirectly using memories which act as data pools shared by processes. A third option distinguishes between synchronous and asynchronous cooperation, where processes either need to stop its activities' until all others are ready or not, respectively.

\subsection{Computational parallelization}

When parallel algorithms are implemented and executed in modern computational environments, different parallel programming models may be applied in a variety of programming environments. Albeit being intertwined (see, for example, [Talbi, 2009]), they represent different facets of parallel implementation from a conceptual perspective. Four (pure) parallel programming models can be distinguished: threads, shared memory, message passing [Diaz et al., 2012, Talbi, 2009] and single-instruction-multiple-data (SIMD). In the thread programming model, lightweight processes

\footnotetext{
${ }^{3}$ Two logics are characterized as "different" even when based on the same methodology (e.g., two tabu searches or genetic algorithms) if they vary in terms of components (e.g., neighborhoods or selection mechanism) or parameter values [Crainic, 2019].
} 
(threads) are executed, where the communication between threads is based on shared addresses. The shared memory programming model, where, too, tasks share a common address space, operates at a higher abstraction level than threads. Today, both the thread and the shared memory model are executed on a multi-core CPU architecture on a single computer node. In contrast, in the message passing programming model the communication between processes is done by sending and receiving messages. Each process has its own address space that is not shared with other processes. This model is designed for execution in computer clusters, where different nodes are connected through high-speed networks. Note that, depending on the particular parallel programming model, parallel executed software parts are labeled differently usually as threads, tasks or processes. Finally, SIMD exploits data parallelism by operating a single instruction on multiple data on a vector processor or array processor. Beyond the pure parallel programming models sketched above, the heterogeneous model General Purpose Computation on Graphics Processing Unit (GPGPU) has received increasing attention (e.g., [Brodtkorb et al., 2013]). GPGPU harnesses the capabilities of multi-core CPUs and many-core GPUs, where threads are executed in parallel on GPU cores and where GPUs can have different levels of shared memory; in this sense, we can speak of heterogeneous systems [Diaz et al., 2012]. Other heterogeneous models are distributed shared memory models and field programmable gate arrays (FPGAs). In modern computing environments, (pure or heterogeneous) parallel programming models are sometimes combined with each other by, e.g., jointly using threads and GPGPU, shared memory and message passing, or threads and message passing [Diaz et al., [2012]. Such approaches are referred to as hybrid models.

Parallel programming environments are related to parallel programming models and comprise languages, libraries, APIs (application programming interfaces) and frameworks.

\subsection{Parallel performance metrics}

The general purpose of parallel computation is to take advantage of increased processing power to solve problems faster or to achieve better solutions. The former goal is a matter of scalability, which is defined as the degree to which it is capable of efficiently utilizing increased computing resources. Performance measures of scalability fall into two main groups: speedup and efficiency. Speedup $S_{p}:=\frac{S}{T_{P}}$ is defined as the ratio of sequential computation time $S$ to parallel computation time $T_{p}$ when the parallel algorithm is executed on $p$ processing units (e.g., cores in a multicore processor architecture). The serial time $S$ can be measured differently, leading to different interpretations of speedup [Barr and Hickman, 1993]: When $S$ refers to the fastest serial time on any serial computer, speedup is denoted as absolute. Alternatively, $S$ may also refer to the time required to solve a problem with the parallel code on one processor. This type of speedup is qualified as relative. When real-time reduction is considered as the primary objective of parallel processing, absolute speedup is the relevant type. While speedup relates serial to parallel times, efficiency $E_{p}:=\frac{S_{p}}{p}$ relates speedup to the number of processing units used. With the definition of efficiency, we can qualify speedup as sublinear speedup $\left(E_{p}<1\right)$, linear speedup $\left(E_{p}=1\right)$, or superlinear speedup $\left(E_{p}>1\right)$. Sublinear speedup is often due to serial parts of a parallel algorithm and several reasons for a nonvanishing serial part can be distinguished. Superlinear speedup can occur, for example, when during the parallel execution of a branch-and-bound algorithm one processor finds a good bound early in the solution process and communicates it to other processors for truncation of their search domains [Barr and Hickman, 1993]. Finally, it should be noticed that while the application of speedup and related efficiency concepts to algorithms which have a "natural" serial version is straightforward, their unmodified application to multi-search algorithms, which are parallel in nature, does not make much sense as no basis of comparison is available.

A second important performance measure in parallel optimization is the solution quality achieved through parallelization. Solution quality can be measured in various ways. When the optimal solution value or a bound of it is known, the relative gap to (the bound of) the optimal value can be determined. A second option is to relate the achieved solution quality with that obtained from sequential versions of the parallelized algorithm (relative improvement). However, this option requires that a sequential version of the parallel algorithm exists in terms of unchanged algorithmic logic and the trajectory through the search space. This is not the case, for example, when cooperative multi-search occurs, which defines a new algorithm due to cooperation. Finally, the solution quality obtained through parallelization may be compared with the quality of the best known solution obtained from any serial implementation (absolute improvement). Overall, the goal of achieving better solutions can be perceived as an issue of effectiveness.

\section{Scope and literature selection process}

The focus of our literature review lies on computational studies of parallel optimization, where physical or virtual parallel computing architectures have been applied to OR problems, such as TSPs, VRPs and FSSPs (flow shop scheduling problems). Due to the interdisciplinary nature of the OR field, such studies are not only found in OR outlets but also in those of many other disciplines, including management science, mathematics, engineering, natural sciences, 
combinations of engineering and natural sciences (such as chemical engineering), computer science, bioinformatics, material science, geology and medicine. While we include outlets of these disciplines in our search (see the succeeding subsection), we would like to stress that the focus of our review lies on studies on OR problems and that it is beyond the scope of this review to identify and classify all articles of parallel optimization addressing problems in related fields or even across all fields (optimization in general). Adopting this view, we exclude from our review, for example, mathematical studies on parallelizing matrix computations or on conjugate gradient methods, computer science studies on load balancing issues in parallel computing environments or on solving hard problems in theoretical computer science (e.g., the subset sum problem), and parallel optimization studies across fields, such as those addressing the effects of migration in parallel evolutionary algorithms. We also exclude works on parallel optimization when their purpose lies in designing or implementing other methodologies, such as simulation, data analysis, data mining, machine learning and artificial intelligence. We further exclude meta optimization (calibrating parameters of optimization models or methodologies). We explicitly acknowledge the importance of these areas but they deserve and need dedicated literature reviews. Finally, from a technological perspective, we also do not consider distributed optimization that makes use of geographically dispersed computers and allows using grids, which comprise networks of many, often thousands or even millions of single computers. This field applies programming models and parallel programming environments that differ from those used in our framework, and it would need a dedicated literature review, too.

Accounting for the previously described scope of our review, we implemented different streams of literature search. A detailed description of the literature search process is provided in the online A Although having implemented different streams of search, we admit that the application of our search procedure does not guarantee to identify all computational studies of parallel optimization in OR and that we may have overlooked studies. However, we are confident to have acquired a body of literature that is sufficiently comprehensive to draw a firm picture of computational parallelization in OR during the decade 2008-2017.

\section{Literature survey}

In this section, we provide a synthesis of the literature published in the decade 2008-2017. We first offer a brief meta analysis, then we analyze the body of literature with regard to which optimization problems have been solved by which (parallelized) algorithms before we present the findings of our literature analysis, structured along optimization algorithms and based upon the framework suggested above. Findings on (i) effectiveness and (ii) parallel programming environments are not presented here because (i) effectiveness results have been reported only rarely and in partially inconsistent ways in the studies of our sample, making comparisons of results difficult, and (ii) parallel programming environments should be considered across algorithms. We discuss both topics in Section 5 With regard to speedup, we qualify it by efficiency when reported in a study. When GPGPU is used as programming model, we only report speedup values without providing the number of parallel processing units or information on efficiency. The reason is that the number of parallel working units (usually GPGPU threads) needs to be interpreted different from that counting other parallel working units (CPU threads, processes) so that efficiency usually being defined as the ratio of speedup and the number of parallel processing units is not applied here. Details on this issue as well as the coding of all studies in our sample are provided in the online B

\subsection{Meta analysis}

Overall, our sample consists of 206 studies, with 164 studies published in 77 different journals, 38 studies published at 36 different workshops, symposiums, conferences or congresses, and four studies published as book chapters. The joint distribution of articles over scientific outlets and years is summarized in Table 1 , which shows that (1) there is no clear temporal development of the numbers of papers published per year, (2) while the number of scholarly outlets (journals, proceedings, etc.) which have published computational studies on parallel optimization in OR is high, only nine outlets have published at least five articles during the decade 2008-2017 and only three outlets (namely, Computers \& Operations Research, European Journal of Operational Research, Journal of Parallel and Distributed Computing) have published more than ten articles in the same period. Overall, this publication landscape does not reveal clear clusters in terms of time or outlet, it rather shows that computational and parallel optimization in OR has been covered permanently (and) distributed over many outlets rooted in different yet related academic disciplines, including $O R$, Computer Science and Engineering. Apparently, this research area is of multidisciplinary relevance.

\subsection{Problem types and parallelized algorithms}

We now describe the identified body of literature from the perspective of problem types and types of parallelized algorithms. Table 2 shows the joint distribution of articles over these two dimensions. We identified problem types by, firstly, coding for each article of our sample the covered problem(s) and, secondly, consolidating problems to 


\begin{tabular}{|c|c|c|c|c|c|c|c|c|c|c|c|}
\hline \multirow{2}{*}{ Outlet } & \multicolumn{10}{|c|}{ Year } & \multirow{2}{*}{ Sum } \\
\hline & 2008 & 2009 & 2010 & 2011 & 2012 & 2013 & 2014 & 2015 & 2016 & 2017 & \\
\hline ASC & & & & 2 & 1 & & 1 & 1 & 1 & 2 & 8 \\
\hline CIE & & & 1 & & & 1 & & 1 & 1 & 1 & 5 \\
\hline COR & 1 & & 1 & & 3 & 2 & 2 & & & 2 & 11 \\
\hline CCPE & & & & & & 1 & & 1 & & 3 & 5 \\
\hline EJOR & & 3 & 1 & & 1 & 1 & 1 & 3 & 2 & 2 & 13 \\
\hline IJOC & 1 & 1 & & 2 & & & & & 1 & 1 & 6 \\
\hline JPDC & 1 & 1 & 1 & 2 & & 4 & 1 & 1 & 1 & & 12 \\
\hline JSC & & & & 1 & & & 1 & & 1 & 2 & 5 \\
\hline PC & 1 & & & 1 & 1 & & & 2 & 2 & 1 & 8 \\
\hline Other journals & 7 & 5 & 5 & 12 & 12 & 11 & 7 & 10 & 12 & 13 & 91 \\
\hline Proceedings & 3 & 5 & 7 & 11 & 4 & 4 & 3 & & 1 & & 37 \\
\hline Book chapters & 2 & 1 & & 1 & 1 & & & & & & 5 \\
\hline Sum & 15 & 15 & 16 & 32 & 22 & 24 & 16 & 18 & 21 & 27 & 206 \\
\hline $\begin{array}{l}\text { ASC: Applied } \\
\text { CIE: Computer } \\
\text { COR: Compute } \\
\text { CCPE: Concur } \\
\text { EJOR: Europe } \\
\text { IJOC: INFORI } \\
\text { JPDC: Journal } \\
\text { JSC: Journal of } \\
\text { PC: Parallel Cc }\end{array}$ & $\begin{array}{l}\text { oft Con } \\
\text { \& Indu } \\
\text { s \& Op } \\
\text { ncy an } \\
\text { Journa } \\
\text { S Journ } \\
\text { f Parall } \\
\text { Superco } \\
\text { aputing }\end{array}$ & $\begin{array}{l}\text { puting } \\
\text { trial Er } \\
\text { rations } \\
\text { Comps } \\
\text { of Ope } \\
1 \text { on Co } \\
l \text { and D } \\
\text { mputins }\end{array}$ & $\begin{array}{l}\text { gineer } \\
\text { Resear } \\
\text { tation- } \\
\text { rationa } \\
\text { mputin } \\
\text { stribut }\end{array}$ & $\begin{array}{l}\mathrm{g} \\
\text { ractice } \\
\text { Resear } \\
\text { Com }\end{array}$ & Expe & ience & & & & & \\
\hline
\end{tabular}

Table 1: Joint distribution of selected articles over scientific outlets and years

problem types widely used in the OR literature ${ }^{4}$ Overall, we identified nine "application-oriented" problem types (AP, FLP, FSSP, GTP, JSSP, KP, MSP, TSP, VRP) and three "mathematically-oriented" problem types (BFP, MILP, SOP) ${ }^{5}$ Adopting this distinction leads to assigning a study that, for example, formulates a TSP as a mixed-integer linear program to the problem class "TSP" rather than to the class "MILP" as it is TSP instances that are focused and not MILP instances in general. Conversely, studies assigned to one of the classes BFP, MILP or SOP explicitly address the related mathematically-oriented problem type and are not necessarily linked to any specific application. We consolidated all problem types for which only very few computational parallelization studies have been published to the category "Other'6

With regard to types of algorithms, we draw on a taxonomy suggested by Talbi [2009], who distinguishes between exact algorithms (e.g., branch-and-bound), problem-specific heuristics (e.g., Lin-Kernighan heuristic for the TSP), single-solution based metaheuristics (e.g., tabu search), and population-based metaheuristics (e.g., genetic algorithms) 7 We extend the taxonomy by adding some algorithm types: hybrid metaheuristics refer to an metaheuristic where parts of a (meta)heuristic $A$ are embedded into a step of a (meta)heuristic $B$; matheuristics refer to the interoperation of metaheuristics and (exact) mathematical programming techniques; multi-search algorithms refers to the combination of several independent search algorithms, which may collaborate or not. Finally, we provide other heuristics as a residual type for those (meta)heuristics which do not fit to any of the aforementioned algorithm types.

It should be noticed that the sums of addressed problem types and parallelized algorithm types shown in Table 2 do not equal the sample size for different reasons: (i) some articles in our sample apply more than one algorithm type to a single problem type and/or investigate more than one optimization problem type; (ii) a few articles do not clearly reveal (from our perspective) the targeted problem or the applied algorithm, or they do not parallelize any algorithm but only the evaluation of the objective function; due to these reasons, we excluded five articles from the presentation in Table 2 Overall, it should be kept in mind that each combination of addressed problem type and parallelized algorithm type is a

\footnotetext{
${ }^{4}$ An example of consolidation is grouping the "multi-depot VRP" and the "VRPs with time windows" to the problem type "VRP".

${ }^{5}$ While application-oriented problem types (e.g., TSP) usually lead to mathematical formulations which have an overall and coherent logic across the components (objective function, constraints, variables, etc.) of a model, "mathematically-oriented" problem types (e.g., MILP) have mathematical formulations where single components have to meet mathematical assumptions (e.g., binary variables, linear terms) without requiring the overall model to refer to a specific application concept.

${ }^{6}$ When an article studies several "other" problem types, we did not count the number of other problem types but coded it as a single appearance of an "other problem type".

${ }^{7}$ The authors also suggest the type approximation algorithms, which we do not use in this review.
} 


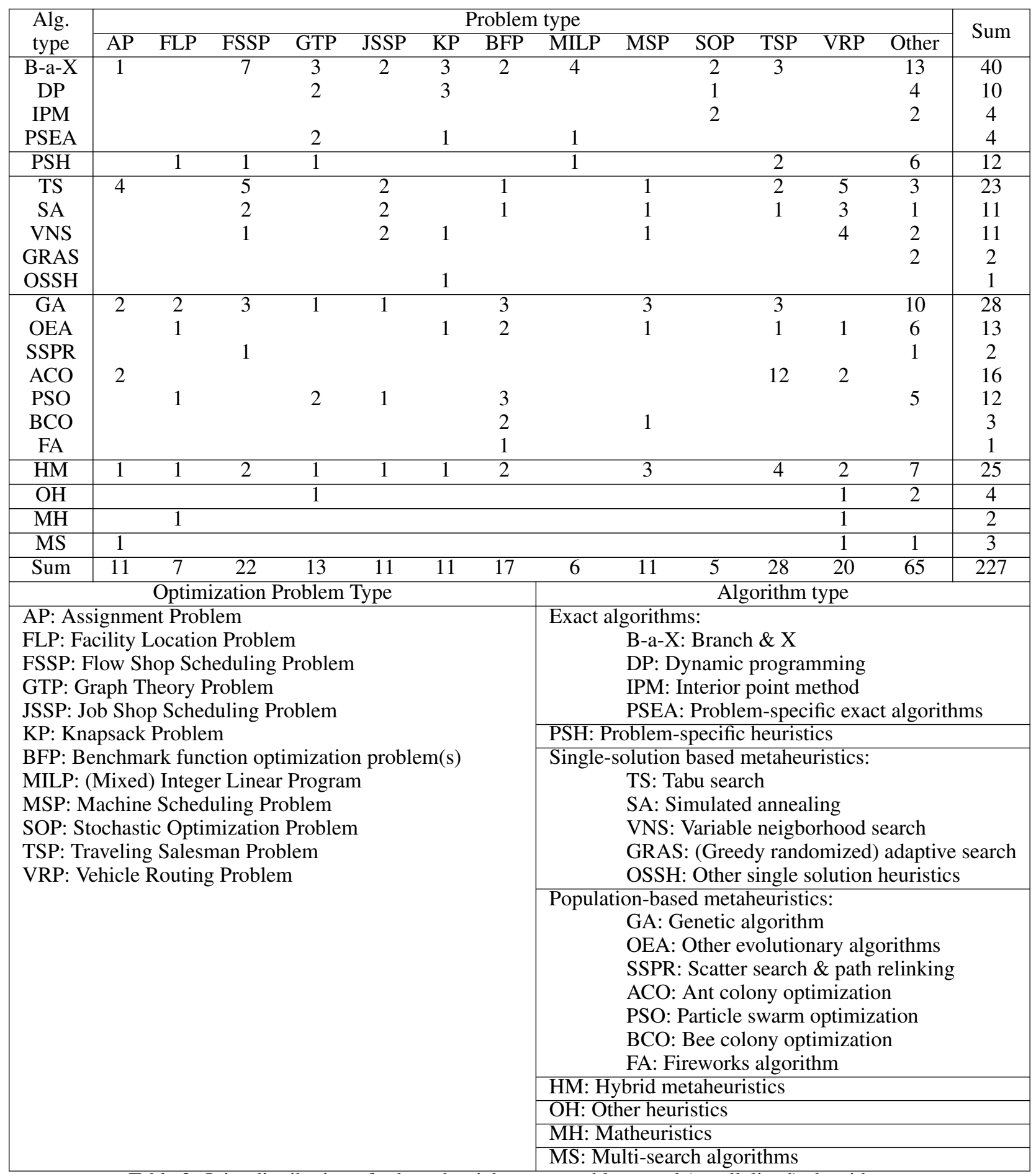

Table 2: Joint distribution of selected articles over problems and (parallelized) algorithms 
"case" of a study, where a single study may have several cases. The perspective on optimization problems addressed in computational parallelization studies shows that a broad range of problem types have been covered. Beyond the 12 problem types highlighted, the residual class of other problem types includes 63 cases, in which computational parallelization has been applied to mostly different problem types. However, we also notice that a set of 12 problem types account for more than 70\% of all cases, with a focus on the TSP, the FSSP and the VRP, which jointly account for more than $30 \%$ of all cases. Similar results are obtained from adopting the algorithmic perspective. While a broad range of exact algorithms and single-solution, population-based and hybrid metaheuristics have been parallelized, only a few algorithm types (branch-and-X ( $\mathrm{X}=$ bound, cut, price, etc.), GAs, hybrid metaheuristics, TS) account for more than $50 \%$ of all cases, with branch-and-X accounting for about $18 \%$. Jointly adopting the problem and algorithmic perspective, again, shows a large diversity but in this case no large clusters occur. Only four combinations (ant colony optimization applied to the TSP, branch-and-X applied to the FSSP, TS applied to the FSSP, TS applied to the VRP) have been covered in at least five cases, but these four combinations account for only about $13 \%$ of all cases.

In the remainder of this section, we present parallel computational optimization studies in OR grouped by algorithm types. An overview over the studies of our sample is given is Table 3 .

Exact algorithms: The majority of studies that apply exact algorithms parallelize branch-and- $\mathrm{X}$ algorithms. These studies analyze a broad range of optimization problems. Almost all adopt domain decomposition as parallelization strategy using a $1 \mathrm{C} / \mathrm{C}$ or $\mathrm{pC} / \mathrm{C}$ scheme with MPSS search differentiation, and most studies which report on the used communication topology apply a (one- or multiple-tier) master-slave approach. These efforts are not surprising as they reflect a straightforward (and traditional) way to parallelize branch-and-X algorithms. In contrast, the landscape of applied parallel programming models is more diverse and includes approaches based on threads, message passing, shared memory and GPGPUs. With regard to the former three models, mostly sublinear or linear speedup has been reported but there are also a few studies [Ponz-Tienda et al., 2017. Borisenko et al., 2011, Galea and Le Cun. 2011] that report superlinear speedup. This speedup can be achieved, for example, when a parallel executed algorithm provides "good" bounds that allow pruning large parts of the search tree at early stages. The use of GPGPUs has shown mixed results in terms of speedup; however, in some cases the reported speedup is substantial (between 76.96 and 170.69) [Chakroun et al., 2013a], which makes GPGPUs highly appealing for parallelizing branch-and-X algorithms. However, it should also be acknowledged that several of these GPGPU studies have reported a high variance of speedup with regard to problem instances solved. Dynamic programming ${ }^{8}$ is the second most often parallelized exact algorithm. Its parallelization in terms of addressed problems is quite diverse. In most cases, low-level is used as parallelization strategy with a 1C/RS scheme and SPSS search differentiation. The landscape of applied communication topologies is quite homogeneous, with almost all studies that report on the applied communication topology drawing on a (oneor multiple-tier) master-slave approach. In contrast, the set of implemented programming models is heterogeneous. Interestingly and in contrast to branch-and-X parallelization, the reported speedups are all sublinear. Studies that use GPGPUs report different ranges of speedup, with one study [Tran, 2010] reporting an exceptionally high speedup in the range of 900-2,500. In addition, we found only a few studies which parallelize the interior point method. All of these studies address stochastic optimization problems, using low-level parallelism in a 1C/RS scheme with SPSS search differentiation, and they achieve sublinear or linear speedup. While all studies apply message passing as parallel programming model, the topologies used differ. Finally, a few exact methods designed for specific optimization problems (the knapsack problem [Li et al., 2015], mixed integer linear programming [Rossbory and Reisner. 2013] and graph theory problems [Kollias et al. [2014, Bozdağ et al., 2008]) have been parallelized. While all four studies show sublinear or linear speedup, the characteristics of algorithmic and computational parallelization are different.

Single-solution based metaheuristics: Single-solution based metaheuristics manipulate and transform a single solution during the search. They can occur in many different forms and their parallelization has been discussed in [Melab et al. 2006, Talbi, 2009]. Parallelization can occur at the solution level, iteration level and algorithmic level. While parallelizing at the solution and iteration level generally corresponds to low-level parallelization with a 1C/RS scheme and SPSS search differentiation, parallelization at the algorithmic level is open to the broad range of parallelization strategies, and process and search control options. Our literature review revealed that mainly three single-solution based metaheuristics have been parallelized: TS, SA and VNS. TS has been applied to a variety of optimization problems. Most studies apply parallelization at the solution or iteration level, thereby adopting low-level parallelization with a $1 \mathrm{C} / \mathrm{RS}$ scheme and SPSS search differentiation and a master-slave communication topology. We found a few exceptions from this algorithmic parallelization pattern; for example, Jin et al. [2012], James et al. [2009], Jin et al. [2014. 2011] adopt cooperative multi-search parallelization of TS, and Dai et al. [2009] implement domain decomposition parallelization of TS. The landscape of applied parallel programming models is quite diverse and includes approaches based on threads, message passing, shared memory, SIMD, and GPGPUs. Speedup results are mixed, including superlinear speedup [Bozejko et al. 2013, Shylo et al. 2011]. The implementation on GPGPUs has shown substantial differences with regard to speedup, reaching values up to 420 [Czapiński, 2013]. The landscape of parallel SA studies,

\footnotetext{
${ }^{8} \mathrm{An}$ introduction into parallel dynamic programming is provided by Almeida et al. [2006].
} 
Parallel computational optimization in operations research

\begin{tabular}{|c|c|}
\hline \multirow{2}{*}{\multicolumn{2}{|c|}{$\begin{array}{l}\text { Algorithm type } \\
\text { Exact algorithms: }\end{array}$}} \\
\hline & \\
\hline Branch \& X & 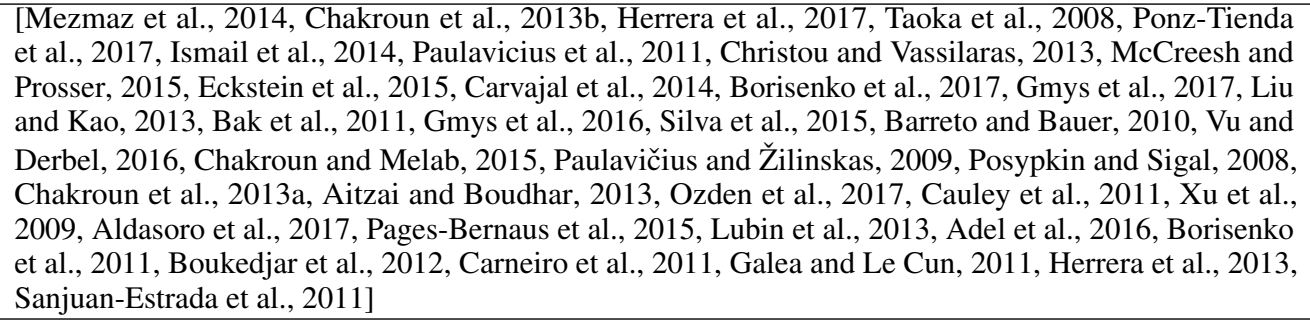 \\
\hline $\begin{array}{l}\text { Dynamic program- } \\
\text { ming }\end{array}$ & 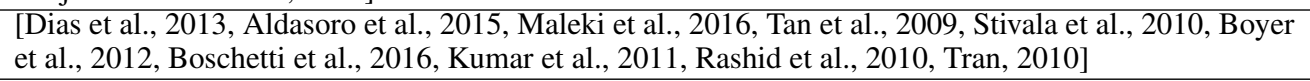 \\
\hline $\begin{array}{ll}\text { Interior } & \text { point } \\
\text { method }\end{array}$ & Huebner et al. 2017, Hong et al. 2010 Lubin et al. 2012 Lucka et al. $20 \overline{1}$ \\
\hline $\begin{array}{l}\text { Problem-specific ex- } \\
\text { act algorithms }\end{array}$ & \begin{tabular}{|l|l|l|l|} 
Li et al. & 2015 & Rossbory and Reisner & 2013 \\
\end{tabular} \\
\hline Problem-specific heuristics & 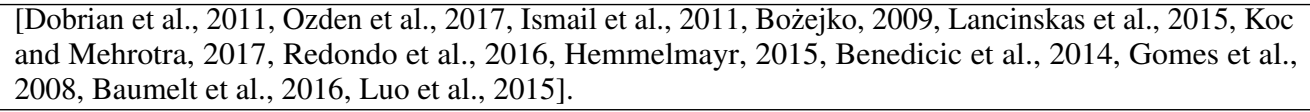 \\
\hline $\begin{array}{l}\text { Single-solution based metaheuristics: } \\
\text { Tabu search }\end{array}$ & 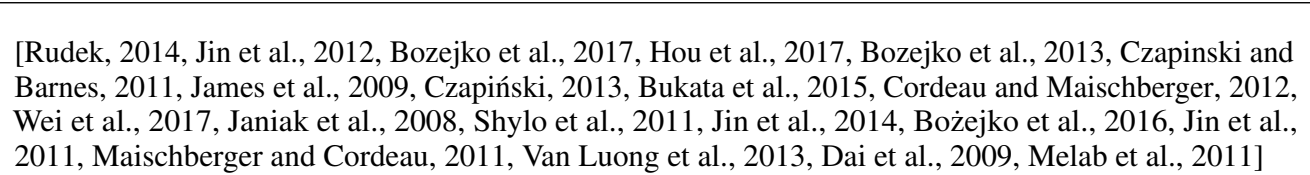 \\
\hline $\begin{array}{l}\text { Simulated anneal- } \\
\text { ing }\end{array}$ & 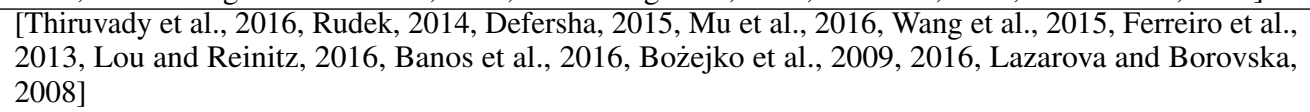 \\
\hline $\begin{array}{l}\text { Variable neigbor- } \\
\text { hrod search }\end{array}$ & 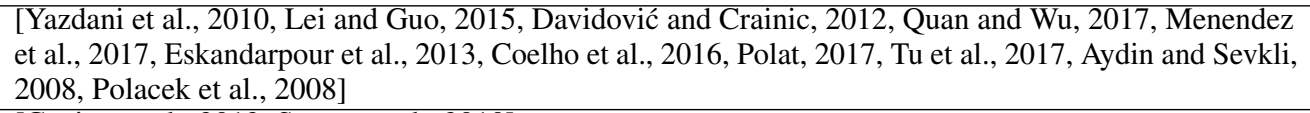 \\
\hline $\begin{array}{ll}\text { (Greedy } & \text { random- } \\
\text { ized) } & \text { adaptive } \\
\text { search } & \end{array}$ & \begin{tabular}{|l|l|l|} 
Caniou et al. 2012 & Santos et al. 2010 \\
\end{tabular} \\
\hline $\begin{array}{l}\text { Other single solu- } \\
\text { tion heuristics }\end{array}$ & [Hifi et al. 2014] \\
\hline Population-based metaheuristics: & \\
\hline Genetic algorithm & 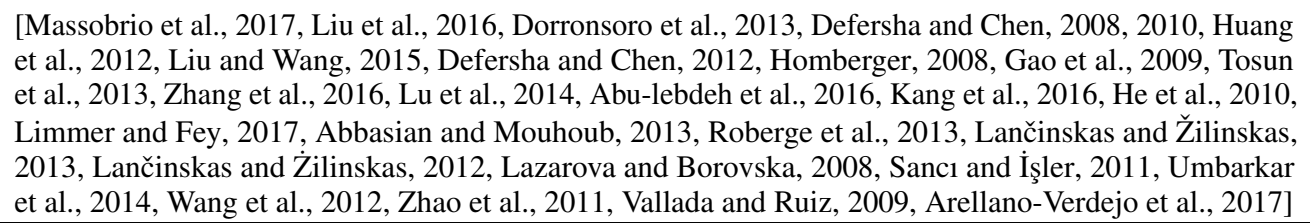 \\
\hline $\begin{array}{l}\text { Other evolutionary } \\
\text { algorithms }\end{array}$ & 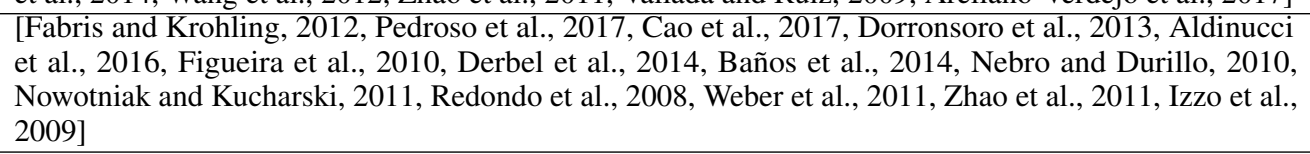 \\
\hline $\begin{array}{l}\text { Scatter search \& } \\
\text { path relinking }\end{array}$ & $\overline{\overline{1 K} \text { Kerkhove and Vanhoucke }}|\overline{2017}, \overline{\text { Bożejko, }}| \overline{2009} \mid$ \\
\hline $\begin{array}{l}\text { Ant colony opti- } \\
\text { mization }\end{array}$ & 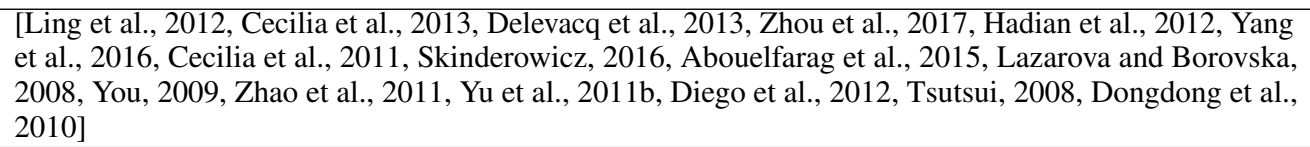 \\
\hline $\begin{array}{l}\text { Particle swarm opti- } \\
\text { mization }\end{array}$ & 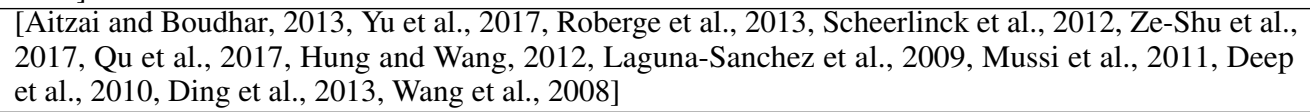 \\
\hline $\begin{array}{l}\text { Bee colony opti- } \\
\text { mization }\end{array}$ & \begin{tabular}{|l|l|l|l|} 
Luo et al. 2014 & Davidovic et al. 2011 Subotic et al. 2011 \\
\end{tabular} \\
\hline $\begin{array}{l}\begin{array}{l}\text { Fireworks } \\
\text { rithm }\end{array} \\
\text { algo- }\end{array}$ & [Ding et al. 2013] \\
\hline Hybrid metaheuristics & 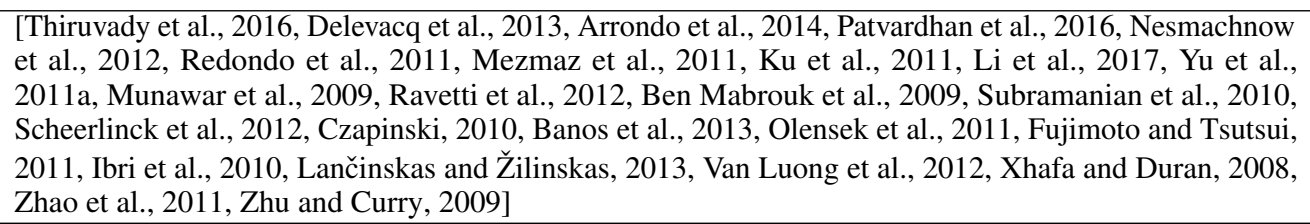 \\
\hline Other heuristics & 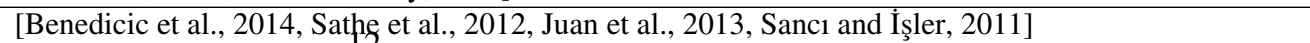 \\
\hline Matheuristics & [Stanojevic et al. $\mid 2015$ Grồr et al. 2011$]$ \\
\hline Multi-search algorithms & 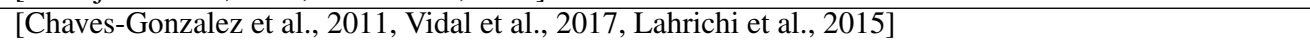 \\
\hline
\end{tabular}

Table 3: Parallel computational optimization studies in OR 
which have also been applied to a variety of optimization problems, is more diverse than that of GA studies. It has been addressed by all four parallelization strategies with varying types of process and search control and with different programming models. In contrast to this heterogeneity, most studies apply a master-slave communication topology. Only a few studies report the achieved speedup, which is mostly sublinear. We found one study [Ferreiro et al., 2013] that parallelizes SA using GPGPU and achieves speedups in the range of about 73.44-269.46. VNS has also been applied to many different problems with all four parallelization strategies and a variety of process and search control variations, communication topologies, and programming models. As in the case of SA, about half of the studies do not report on speedup and those which do report sublinear speedup, with the exception of Polacek et al. [2008], who achieve linear speedup. One study uses GPGPU [Coelho et al. [2016] and achieves a speedup in the range of 0.93-14.49. Additionally, we found two studies [Caniou et al., 2012, Santos et al., 2010] that parallelize (greedy randomized) adaptive search and one study [Hifi et al. [2014] that parallelizes large neighborhood search (subsumed under "other single solution heuristic (OSSH)" in Table 2).

Population-based metaheuristics: In contrast to single-solution based metaheuristics, in population-based algorithms a whole population of solutions is evolved. Most prominent classes of population-based metaheuristics include evolutionary algorithms, scatter search and path relinking, swarm intelligence algorithms, and bee colony optimization [Talbi, 2009]. When population-based algorithms are parallelized, we distinguish three models which, albeit having been suggested originally for evolutionary algorithms in general and GAs in particular [Alba and Tomassini, 2002. Talbi, 2009, Agrawal and Mathew, 2004, Melab et al., 2006, Cantú-Paz, 2005, Luque et al., 2005], can be applied to other classes of population-based algorithms as well: global, island (with or without migration), and cellular model. In the global model, parallel techniques are used to speed up the operation of the algorithm without changing the basic operation of the sequential version. When the evaluation of the whole population is done in parallel, parallelism occurs at the iteration level; when the algorithm evaluates a single individuum in parallel, parallelism occurs at the solution level. In both cases, low-level parallelization applies. Island models typically run (identical or different) serial population-based algorithm on subpopulations to avoid getting stuck in local optima of the search space. If individuals can be transmitted between subpopulations, the island model is also referred to as migration model; however, island models can also occur without migration. While in the former case, migration usually leads to a cooperative multi-search, the latter case generally corresponds to independent multi-search parallelization. The cellular model may be seen as a special case of the island model where an island is composed of a single individual. It should be noted that the models may be applied jointly (Cantú-Paz [2005], for example, describes such model combinations for GAs).

Evolutionary algorithms belong to the types of algorithms that have attracted substantial parallelization efforts. A good overview of the diversity with which combinations of different parallelization strategies and programming models can be applied to evolutionary algorithms is provided by Limmer and Fey [2017]. In our sample, we found a focus on GAs as a particular subclass of evolutionary algorithm; we subsume all evolutionary algorithms other than GAs under the residual subclass"other evolutionary algorithms". GAs have been parallelized for a variety of optimization problems. Most of the studies adopt the island model with migration (cooperative multi-search) with a pC/RS scheme and MPSS or MPDS search differentiation. Only a few studies use the island model without migration (independent multi-search) with a pC/RS scheme and MPSS search differentiation, or the global model (low-level) with a 1C/RS scheme and SPSS search differentiation. Interestingly, all but one study [Vallada and Ruiz, 2009] apply synchronous communication. In the presence of the island model, a diversity of communication topologies has been applied with mostly message passing being used as programming model. In contrast, when the global model is applied, threads or GPGPU are drawn upon and mostly the master-slave topology is implemented. The described correlation between the parallelization strategy and the parallel programming model is not surprising as the communication between (a usually moderate number of) islands through exchanging messages is appealing while the processing of (a usually large number of) individuals in a global population through (an often large number of) threads executed on a CPU or GPGPU seems appropriate. Only about half of the 27 GA studies that we found report speedup values. Speedup results are overall mixed, including superlinear speedup [Homberger, 2008, Abu-lebdeh et al., 2016]. The application of GPGPUs has led to homogeneous results, with a maximum speedup of about 33 [Wang et al.|2012]. Evolutionary algorithms other than GAs, such as differential evolution or immune algorithm, have been applied to a variety of optimization problems. Almost all of these studies adopt the island model with migration (cooperative multi-search) with a pC/RS scheme and MPSS or MPDS search differentiation. We found only two studies [Baños et al., 2014, Izzo et al., 2009] which report an asynchronous communication. We identified no pattern regarding the applied communication topology and programming model.

Swarm intelligence algorithms are inspired from the collective behavior of species such as ants, fish and birds. Subclasses of swarm intelligence algorithms for which we found parallelization studies are ant colony optimization (including ant colony systems and "MAX-MIN Ant Systems" [Dorigo and Stützle, 2004]), particle swarm optimization, and fireworks algorithms. Parallelization strategies of ant colony optimization can be classified according to the above mentioned three strategies of parallelizing population-based metaheuristics; i.e., global, island or cellular model. Here, we follow the 
suggestion of Randall and Lewis [2002] to distinguish the parallel evaluation of solution elements, parallel ant colonies (independent or interacting) and parallel ants. These strategies are specializations of the global model, island model (without or with migration), and cellular model, respectively, of population-based metaheuristics. Interestingly, most of the parallelization studies using ant colony optimization have addressed the TSP. VRPs [Yu et al., 2011b, Diego et al. 2012] and assignment problems [Tsutsui 2008, Dongdong et al. 2010] have been solved by two studies each. Almost all studies use parallel ants or multiple ant colonies but, overall, the studies vary regarding parallelization strategies, process and search control, communication topologies and programming models. Those studies which qualify the achieved speedups, report sublinear speedups. The speedup achieved through GPGPU parallelization goes up to 25 . Particle swarm optimization has been applied to solve a diverse set of optimization problems. Most of the parallelization studies make use of the global or island model, realized as low-level or cooperative multi-search parallelization, respectively, with a master-slave communication topology. The process and search control implementations differ, with only one study [Wang et al., 2008] reporting asynchronous communication. Mostly message passing and GPGPU are used as parallel programming model. Speedups achieved on GPGPU go up to about 190; studies not using the GPGPU model either do not report speedup values or show an overall diverse picture. In addition, we identified one study [Ding et al. 2013] that applies a fireworks algorithm.

Other population-based metaheuristics: We identified five studies that parallelize population-based metaheuristics other than evolutionary algorithms and swarm intelligence algorithms, namely scatter search and path relinking [Kerkhove and Vanhoucke, 2017, Bożejko, 2009], and bee colony optimization [Luo et al., 2014, Davidovic et al., 2011, Subotic et al., 2011]. Addressed problems, algorithmic and computational parallelization characteristics as well as efficiency results (where reported) are quite diverse.

Hybrid metaheuristics: Hybrid metaheuristics are joint applications of several (meta)heuristics [Talbi 2009. Crainic. 2019]. They are "appropriate candidates" for the application of a(n) (independent or cooperative) multi-search strategy. A diverse set of optimization problems has been investigated with parallel hybrid metaheuristics. The combinations of (meta)heuristics include ant colony optimization and local search, GAs and local search, GAs and SA, and GAs and TS, among others. Due to the diverse set of combined (meta)heuristics, unsurprisingly, the studies differ substantially with regard to addressed problems, parallelization strategies, process and search and control, communication topologies and parallel programming models. Although none of these studies report a superlinear speedup, Zhu and Curry [2009] reports an achieved speedup of 403.91 when parallelizing a combination of ant colony optimization and pattern search with a GPGPU-based implementation.

Problem-specific heuristics, other heuristics, matheuristics, and multi-search algorithms: Problem-specific heuristics have been parallelized for a variety of optimization problems, including a graph theory problem [Dobrian et al. 2011], TSPs [Ozden et al., 2017., Ismail et al., 2011], a FSSP [Bożejko, 2009], a facility location problem [Lancinskas et al., 2015], a mixed integer linear program [Koc and Mehrotra, 2017], and several other problems |Redondo et al., 2016, Hemmelmayr. 2015, Benedicic et al. |2014, Gomes et al., 2008, Baumelt et al., 2016, Luo et al. 2015. . We found four studies which parallelize heuristics that differ from all types described above: an agent-based heuristic [Benedicic et al., 2014], an auction-based heuristic [Sathe et al., 2012], a Monte Carlo simulation inside a heuristic-randomization process [Juan et al. 2013], and a random search algorithm [Sanc1 and İşler, 2011]. We found two studies which parallelize matheuristics [Stanojevic et al., 2015, Groer et al., 2011] and three studies which suggest multi-search algorithms [Chaves-Gonzalez et al., 2011, Vidal et al.|2017, Lahrichi et al., 2015]. Due to the diverse nature of the aforementioned studies, we do not look for patterns in algorithmic parallelization, computational parallelization and scalability results.

\section{Research directions}

Based on the analysis of the identified literature published in the covered period (2008-2017), we subsequently suggest some research directions which may help (re)focusing on those areas that did not get much attention or were even neglected during the focused period. We would like to note that the observation of the absence or rareness of certain types of studies primarily refers to the aforementioned period. Work published prior to this period and surveys published earlier than this review (see Section 1) have addressed some of the "white spots" in research identified for the aforementioned period, which calls for $r e$-focusing on related research paths.

\subsection{Publication landscape and overall prospective research}

The analysis of publication data reveals that computational and parallel optimization in OR has been steadily attractive for many journals and conferences not only in the OR field but also in various neighbor disciplines. This broad interest is also reflected in the diverse landscape of which optimization problems have been solved by which (parallelized) algorithms. While this diversity shows the large relevance and broad applicability of computational parallelization in 
optimization, a closer look also reveals that the landscape is still fragmented despite the algorithmic accumulation of branch-and-X, GAs and TS studies and the problem accumulation of FSSPs, TSPs and VRPs. This makes it difficult to analyze which combinations of problems and algorithms are promising for parallelization and how the algorithmic and computational parallelization should be designed. It should be noted that in the presence of a broad scope of problems and algorithms in parallel optimization, the number of approximately 200 studies published in ten years is relatively low. Future research and education can benefit from fostering (knowledge on how to conduct) computational studies in parallel optimization to overcome the limitations imposed by fragmentation (recommendations 1a and 1b in Table 47.

\subsection{Object of parallelization}

From the algorithmic perspective, branch-and-X algorithms represent the largest cluster of computational parallelization studies. In a few studies, this parallelization has even led to superlinear speedup but in most cases "only" (sub)linear speedups have been achieved. Future research should shed more light on how to achieve superlinear speedups (recommendation 2a). With regard to dynamic programming, which is the second most often analyzed type of exact algorithms, the (sublinear) speedup achievements are less promising (see recommendation $2 \mathrm{~b}$ ). Again, our subsample of dynamic programming studies and their coding can serve as a basis for future investigations on more efficient dynamic programming parallelization, in particular on how to achieve superlinear speedup. We extend this recommendation to future research on parallelization of Lagrangean decomposition, which is - as dynamic programming - another methodology often used in the important field of stochastic optimization but which has hardly been parallelized. Parallelization efforts with regard to interior point methods are hardly existent, which asks for more research in this regard (recommendation 2c).

Among single-solution based metaheuristics, three metaheuristics have received particular attention regarding parallelization: TS, SA and VNS. For TS, speedup results are mixed, including two studies that report superlinear speedups, and the implementation on GPGPUs has shown substantial differences with regard to speedup. Future research should analyze this heterogeneous picture (recommendation 2d). With regard to SA and VNS, not much can be said on efficiency as, unfortunately, many studies do not report achieved speedups (see recommendation 2e). Beyond the aforementioned metaheuristics, other single-solution based metaheuristics, including greedy randomized adaptive search, guided local search, fast local search, and iterated local search [Gendreau et al., 2010, 2019], have not received much attention with regard to parallelization, which points to further research opportunities (recommendation $2 \mathrm{f}$ ).

With regard to population-based metaheuristics, GAs are the most often parallelized type of algorithm. However, only a few studies provide speedup values, some of them reporting superlinear speedups. While these achievements are promising, not much knowledge about the factors that lead to superlinear speedup (see recommendation $2 \mathrm{~g}$ ) has been developed. Furthermore, parallelization results for GAs as well as other evolutionary algorithms are mainly based on synchronous communication so that not much is known about the potential of applying asynchronous communication (recommendation $2 \mathrm{~h}$ ). The second and third most often parallelized type of population-based metaheuristics are ant colony optimization and particle swarm optimization, respectively. With regard to ant colony optimization, achieved speedups are not very promising and mostly limited to applications to the TSP. Regarding particle swarm optimization, speedup results are quite mixed, with a promising speedup value of about 190 reported when using the GPGPU model. These results show that further research on parallelizing ant colony optimization and particle swarm optimization is recommendable (recommendation 2i). Analogously to single-solution based metaheuristics, some algorithms of population-based methaheuristics, including SSPR, BCO and FA, have not received much attention, which shows avenues for further research (recommendation $2 \mathrm{j}$ ).

Interestingly, we found only very few research on the parallelization of mat-heuristics. We believe that the parallelization of both of its' elements, metaheuristic components and exact mathematical programming techniques, are promising areas of future research (recommendation $2 \mathrm{k}$ ).

Similarly few attention has been attracted by multi-search algorithms, which offer a straightforward parallelization approach through parallelizing the execution of independent search algorithms involved in multi-search. We consider this research stream, in particular cooperative multi-search algorithms, to be highly relevant for future research on parallelization (recommendation 21).

Beyond the previously identified algorithmic research directions, future research should also adopt problem-specific perspectives (recommendation $2 \mathrm{~m}$ ).

\subsection{Algorithmic parallelization and computational parallelization}

The algorithmic parallelization in the studies of our sample has drawn on all four (pure) parallelization strategies and on combinations of pure strategies. Low-level parallelization is the most often implemented strategy, with 83 out of 206 
studies having used this type of parallelism. The process and search control is usually a 1C/RS scheme with SPSS search differentiation. Most studies which use low-level parallelism apply a master-slave communication topology, which is a straightforward approach. However, there are several exceptions, including fully-connected meshs (e.g., [Huebner et al. [2017]) and trees (e.g., [Tan et al., 2009]). It would be useful to know under which conditions communication topologies other than the master-slave topology are advantageous for low-level parallelization (recommendation 3a). Interestingly, even for low-level parallelism a diverse set of parallel programming models and environments have been used, including message passing. This is a bit surprising as message passing is generally applied for the communication between "'heavy weight processes" executed on different computing nodes.

Domain decomposition as parallelization strategy occurs in 56 studies, with most of them parallelizing branch-and-X algorithms, which can be parallelized straightforward by decomposition. Regarding control cardinality, we found 1C and $\mathrm{pC}$ control modes applied similarly often. However, control and communication mostly follows an asynchronous, collegial scheme with no knowledge being exchanged between parallel processes; the used search differentiation is largely MPSS. Future research may explore opportunities that knowledge-based communication offer (recommendation $3 b)$.

Independent multi-search as a parallelization strategy has been applied in only 18 studies, in contrast to cooperative multi-search, which has been implemented in 72 studies. This trend is encouraging as the potential of exchanging information between parallel processes in order to jointly achieve better solutions in less time has thereby been acknowledged by researchers. The vast majority of all studies which apply (independent or cooperative) multi-search uses a (synchronous) rigid synchronization (type "RS"); we identified only four studies [Groer et al., 2011, Bukata et al. 2015, Jin et al. 2014, Lahrichi et al. 2015] which make use of knowledge-based communication. Future research should foster the exploration of knowledge-based communication when multi-search is applied (recommendation 3c). Parallelization strategies can be combined to exploit complementary ways of parallelizations. For example, low-level and domain decomposition parallelism have been jointly applied to branch-and-X algorithms [Vu and Derbel, 2016, Adel] et al. 2016] and to dynamic programming [Maleki et al., 2016], and low-level and multi-search parallelism to genetic algorithms [Abbasian and Mouhoub, 2013,|Munawar et al.|[2009]. In total, we found eight studies which apply such combinations. Future research should more intensively tap the potential that joint applications of different parallelization strategies offer (recommendation 3d). Finally, different parallelization strategies can be applied (separately) to the same algorithm and problem in order to compare their effectiveness and scalability and to determine most appropriate and inappropriate parallelizations. Although we identified as many as 21 studies which follow this path, we encourage scholars to intensify research in this regard (recommendation 3e).

A broad range of different communication topologies has been applied, with master-slave being the most often used topology. The appropriateness of a communication topology needs to be linked to the particular algorithm and the applied parallelization strategy so that no general recommendations are appropriate. However, in the sample of computational studies we found only a few studies (e.g., |Mezmaz et al. 2014. Herrera et al. 2013, Rashid et al. 2010, Aydin and Sevkli [2008]) that have implemented more than one topology for one parallelization strategy of a particular algorithm. This low number calls for more studies that investigate multiple topologies for particular combinations of algorithms and parallelization strategies (recommendation $3 \mathrm{f}$ ).

The parallel implementation of optimization algorithms has exploited overall a rich set of programming models and modern programming environments, including low-level threads (Java threads and POSIX threads), shared memory (mainly OpenMP), message passing (mainly MPI), and GPGPUs (mainly CUDA-based). In addition, also hybrid programming models, including message passing and shared memory, shared memory and GPGPU, threads and GPGPU, and message passing and threads, have been used in a few studies. Other programming models, such as SIMD, have only rarely been used. We found several studies which provide either no or incomplete information on the used parallel programming model(s). We recommend that studies report on the programming model and programming environment used for their parallelization (recommendation $3 \mathrm{~g}$ ).

Only a few studies report on their (re-)use of software frameworks for parallelization, such as ParadisEO [INRIA. n.d.] for parallel and distributed metaheuristics or Bob++ [Djerrah et al. 2006] for branch and bound parallelization. Reasons for not drawing on such frameworks can be manifold. Scholars may deliberately decide to not make use of them due to the inappropriateness of frameworks for their implementation case or due to too time-consuming efforts to get acquainted with the frameworks. Or, scholars are not aware of the existence of such frameworks. Either way, the development, propagation and use of re-usable software frameworks can substantially reduce the tedious and error-prone implementation of parallel optimization code (see recommendation $3 \mathrm{~h}$ ). 


\subsection{Performance of parallelization}

Scalability is essential regarding the appropriateness of a parallel implementation of an optimization algorithm. Interestingly, in 70 out of 206 studies speedup values are not (completely) reported or speedup is interpreted different from how it is usually done (see Section 2.4); for example, some studies determine the speedup by executing the serial and the parallel code on different hardware, resulting in speedup values that are challenging to interpret. Other studies determine the speedup only of parts of an algorithm or use another parallel implementation as base (see B for more details). In such cases, speedup values are hardly comparable with those of other studies and, thus, limit the usefulness of scalability analysis (see recommendation 4a).

But even in case speedup is provided, comparisons with other studies need to be done carefully for several reasons: First, scalability results are difficult to compare with those of other studies when technological characteristics of parallel working units (or even of hardware environments) differ. For example, threads at the software level need to be distinguished from threads at the hardware level (hyperthreading), and MPI processes executed on different physical nodes may perform different from those executed on different cores on the same physical node. Second, values of weak speedup need to be distinguished from those of relative speedup (see Section 2.4. A list of issues related to speedup comparison is provided in $B$ We condense our suggestions in recommendation $4 \mathrm{~b}$.

We analyzed the studies in our sample with regard to how many parallel working units (threads or processes) have been used, which we refer to as range of parallelization. The number of parallel threads executed on a CPU has been mostly not above 32 and it reaches its maximum at 128. When message passing is used on one or several nodes, the number of parallel processes units has in most cases not exceeded 256 and it has reached its maximum at 8,192. Hybrid approaches mostly use up to 1,024 parallel units, with the maximum number having been 2,048. Overall, the range of parallelization is quite limited compared to the number of parallel units that are available in modern parallel computing environments (see recommendation $4 \mathrm{c}$ ).

Our analysis of how studies in the literature have considered the effectiveness of parallelization (to obtain better solutions) showed that many studies do not analyze this category of performance and that those studies which provide effectiveness results use many different ways to report these. They apply different stop criteria (numbers of iterations, wall time, number of function evaluations, combinations of these criteria, etc.) and different evaluation criteria (objective value, relative gap to the best (known) solution value, numbers of instances solved to optimality, relative improvements, etc.), and often do not make the applied stop criteria explicit, which makes it difficult to assess parallel implementations and to compare studies with regard to effectiveness (see recommendation $4 \mathrm{~d}$ ).

\subsection{Presentation of studies}

Finally, having reviewed more than two hundreds of parallelization studies, we found that studies differ substantially in the way how information on parallelization is provided, to what extent information is made explicit, and in which section(s) of the paper which information on parallelization is provided. This heterogeneity may reflect different practices in various subfields and journals, and it not advisable to recommend any standardization in this regard. However, in several studies we found information on parallelization being reported incomplete, intransparent or distributed, which can make it tedious to fully understand the applied parallelization. The framework suggested in this paper may help to mitigate these issues when researchers adopt it and describe how it applies to their studies (recommendation 5).

\section{Conclusion}

This invited review suggests a new integrative framework for parallel computational optimization. It integrates the perspectives on parallel optimization found in the disciplines of OR and computer science, and it distinguishes four levels: i) object of parallelization, ii) algorithmic parallelization, iii) computational parallization, and iv) performance of parallelization. We apply this framework to synthesize the body of literature (206 studies published between 2008 and 2017) of parallel computational optimization in OR. It should be noticed that the applicability of the suggested framework is not limited to the OR field. Finally, we suggest several bundles of research recommendations for parallel computational optimization in OR, with the recommendations grouped along the layers of the suggested framework. 
Publication landscape and overall prospective research

\begin{tabular}{|c|c|}
\hline \multicolumn{2}{|r|}{ Publication landscape and overall prospective research } \\
\hline 1a & $\begin{array}{l}\text { Implementation of dedicated (tracks at) workshops and conferences and publication of edited books, such as Alba, 2005, Talbi 2006, } \\
\text { and of special issues in journals }\end{array}$ \\
\hline $1 \mathrm{~b}$ & Integration of parallel optimization and its application in modern parallel computing environments in curricula of OR education \\
\hline \multicolumn{2}{|r|}{ Object of parallelization } \\
\hline $2 \mathrm{~b}$ & $\begin{array}{l}\text { Identification of ways to make parallelization of dynamic programming and of Lagrangean decomposition more efficient and to achieve } \\
\text { superlinear speedup. Our subsample of dynamic programming studies and their coding can serve as a basis for future investigations. }\end{array}$ \\
\hline $2 \mathrm{e}$ & Amplification of scalability analysis with regard to parallelizations of SA and VNS. \\
\hline $2 \mathrm{f}$ & $\begin{array}{l}\text { Extension of parallelization efforts to a more comprehensive set of single-solution based metaheuristics, including greedy randomized } \\
\text { adaptive search, guided local search, fast local search, and iterated local search. }\end{array}$ \\
\hline $2 \mathrm{~g}$ & Identification of those factors that drive superlinear speedup when parallelizing GAs. \\
\hline $2 \mathrm{~h}$ & Application of asynchronous communication to genetic algorithms and other evolutionary algorithms. \\
\hline $2 \mathrm{i}$ & Amplification of parallelization efforts with regard to ant colony optimization and particle swarm optimization. \\
\hline $2 \mathrm{~m}$ & $\begin{array}{l}\text { Adoption of problem-specific perspectives by analyzing which parallelization efforts (algorithms, parallel algorithm designs, parallel } \\
\text { implementations) lead to which performance for a particular optimization problem. From Table } 2 \text { it can be seen that, in particular, FSSPs, } \\
\text { TSPs, and VRPs have attracted fairly high number of parallelization studies that can be used for further analysis. }\end{array}$ \\
\hline \multicolumn{2}{|r|}{$\begin{array}{l}\text { Algorithmic parallelization and computational parallelization } \\
\end{array}$} \\
\hline $3 \mathrm{a}$ & $\begin{array}{l}\text { Identification of conditions under which communication topologies other than the master-slave topology are advantageous for low-level } \\
\text { parallelization. }\end{array}$ \\
\hline $3 \mathrm{~b}$ & Exploration of opportunities that knowledge-based communication offers in the case of domain decomposition. \\
\hline $3 \mathrm{c}$ & Exploration of knowledge-based communication when multi-search parallelism is applied. \\
\hline $3 \mathrm{~d}$ & Tapping the potential that joint applications of different parallelization strategies offer. \\
\hline $3 \mathrm{e}$ & $\begin{array}{l}\text { Comparisons of effects that different parallelization strategies have when applied to a particular algorithm and problem in order to } \\
\text { determine (in)appropriate parallelization strategies in this case. }\end{array}$ \\
\hline $3 f$ & $\begin{array}{l}\text { Investigation of multiple strategies and/or multiple topologies for a particular algorithm in order to compare the performance of these } \\
\text { alternatives. }\end{array}$ \\
\hline $4 \mathrm{~d}$ & Amplification of research on effectiveness of computational parallelization and documentation of applied stop and evaluation criteria. \\
\hline \multicolumn{2}{|r|}{ Presentation of studies } \\
\hline 5 & mpleteness, intransparency and distributed provision \\
\hline
\end{tabular}

Table 4: Recommendations for future research on parallel computational optimization in OR 


\section{References}

R. Abbasian and M. Mouhoub. A hierarchical parallel genetic approach for the graph coloring problem. Applied Intelligence, 39(3):510-528, Oct. 2013. ISSN 0924-669X.

A. A. Abouelfarag, W. M. Aly, and A. G. Elbialy. Performance analysis and tuning for parallelization of ant colony optimization by using OpenMP. In Proceedings of the 14th IFIP TC 8 International Conference on Computer Information Systems and Industrial Management, pages 73-85, 2015.

G. Abu-lebdeh, H. Chen, and M. Ghanim. Improving performance of genetic algorithms for transportation systems: case of parallel genetic algorithms. Journal of Infrastructure Systems, 22(4), Dec. 2016. ISSN 1076-0342. doi: 10.1061/(ASCE)IS.1943-555X.0000206.

P. Adamidis. Review of parallel genetic algorithms bibliography. Aristotle Univ. Thessaloniki, Thessaloniki, Greece, Tech. Rep, 1994.

D. Adel, A. Bendjoudi, D. El Baz, and A. Z. Abdelhakim. GPU-based two level parallel B\&B for the blocking job shop scheduling problem. Applied Soft Computing, pages 747-755, 2016.

J. Agrawal and T. V. Mathew. Transit route network design using parallel genetic algorithm. Journal of Computing in Civil Engineering, 18(3):248-256, July 2004. ISSN 0887-3801.

A. Aitzai and M. Boudhar. Parallel branch-and-bound and parallel PSO algorithms for job shop scheduling problem with blocking. International Journal of Operational Research, 16(1):14-37, 2013.

E. Alba. Parallel metaheuristics: A new class of algorithms, volume 47. John Wiley \& Sons, 2005.

E. Alba and G. Luque. Measuring the performance of parallel metaheuristics, chapter 2, pages 43-62. John Wiley \& Sons, Inc., Hoboken, New Jersey, 2005. ISBN 9780471739388.

E. Alba and M. Tomassini. Parallelism and evolutionary algorithms. IEEE Transactions on Evolutionary Computation, 6(5):443-462, Oct. 2002. ISSN 1089-778X.

E. Alba and J. M. Troya. A survey of parallel distributed genetic algorithms. Complexity, 4(4):31-52, 1999.

E. Alba, E.-G. Talbi, G. Luque, and N. Melab. Metaheuristics and parallelism, chapter 4, pages 79-103. John Wiley \& Sons, Inc., Hoboken, New Jersey, 2005. ISBN 9780471739388.

E. Alba, G. Luque, and S. Nesmachnow. Parallel metaheuristics: recent advances and new trends. International Transactions in Operational Research, 20(1):1-48, 2013.

U. Aldasoro, L. F. Escudero, M. Merino, J. F. Monge, and G. Perez. On parallelization of a stochastic dynamic programming algorithm for solving large-scale mixed 0-1 problems under uncertainty. Top, 23(3):703-742, Oct. 2015. ISSN 1134-5764.

U. Aldasoro, L. F. Escudero, M. Merino, and G. Perez. A parallel branch-and-fix coordination based matheuristic algorithm for solving large sized multistage stochastic mixed 0-1 problems. European Journal of Operational Research, 258(2):590-606, Apr. 2017. ISSN 0377-2217. doi: 10.1016/j.ejor.2016.08.072.

M. Aldinucci, S. Campa, M. Danelutto, P. Kilpatrick, and M. Torquati. Pool evolution: a parallel pattern for evolutionary and symbolic computing. International Journal of Parallel Programming, 44(3, SI):531-551, June 2016. ISSN 0885-7458. doi: 10.1007/s10766-015-0358-5.

F. Almeida, D. González, and I. Peláez. Parallel dynamic programming, chapter 2, pages 29-51. John Wiley \& Sons, Inc., Hoboken, New Jersey., 2006. ISBN 9780470053928.

J. Arellano-Verdejo, S. Godoy-Calderon, F. Alonso-Pecina, A. Guzman Arenas, and M. Antonio Cruz-Chavez. A new efficient entropy population-merging parallel model for evolutionary algorithms. International Journal of Computational Intelligence Systems, 10(1):1186-1197, Jan. 2017. ISSN 1875-6891. doi: 10.2991/ijcis.10.1.78.

A. G. Arrondo, J. L. Redondo, J. Fernandez, and P. M. Ortigosa. Solving a leader-follower facility problem via parallel evolutionary approaches. Journal of Supercomputing, 70(2):600-611, Nov. 2014. ISSN 0920-8542. doi: 10.1007/s11227-014-1106-0.

M. E. Aydin and M. Sevkli. Sequential and parallel variable neighborhood search algorithms for job shop scheduling. In Metaheuristics for scheduling in industrial and manufacturing applications, pages 125-144. Springer, 2008.

M. E. Aydin and V. Yigit. Parallel simulated annealing, chapter 12, pages 267-287. John Wiley \& Sons, Inc., Hoboken, New Jersey., 2005. ISBN 9780471739388.

S. Bak, J. Blazewicz, G. Pawlak, M. Plaza, E. K. Burke, and G. Kendall. A parallel branch-and-bound approach to the rectangular guillotine strip cutting problem. INFORMS Journal on Computing, 23(1):15-25, WIN 2011. ISSN 1091-9856. doi: 10.1287/ijoc.1100.0394. 
R. Banos, J. Ortega, C. Gil, A. Fernandez, and F. De Toro. A simulated annealing-based parallel multi-objective approach to vehicle routing problems with time windows. Expert Systems with Applications, 40(5):1696-1707, Apr. 2013. ISSN 0957-4174.

R. Baños, J. Ortega, and C. Gil. Hybrid MPI/OpenMP parallel evolutionary algorithms for vehicle routing problems. In Proceedings of the 18th International Conference on the Applications of Evolutionary Computation, pages 653-664. Springer-Verlag Berlin, 2014.

R. Banos, J. Ortega, C. Gil, F. De Toro, and M. G. Montoya. Analysis of OpenMP and MPI implementations of meta-heuristics for vehicle routing problems. Applied Soft Computing, 43:262-275, June 2016. ISSN 1568-4946.

R. S. Barr and B. L. Hickman. Reporting computational experiments with parallel algorithms: Issues, measures, and experts' opinions. ORSA Journal on Computing, 5(1):2-18, 1993.

L. Barreto and M. Bauer. Parallel branch and bound algorithm - a comparison between serial, OpenMP and MPI implementations. Journal of Physics: Conference Series, 256(1):012018, 2010. URL http://stacks.iop.org/ $1742-6596 / 256 / i=1 / a=012018$

Z. Baumelt, J. Dvorak, P. Sucha, and Z. Hanzalek. A novel approach for nurse rerostering based on a parallel algorithm. European Journal of Operational Research, 251(2):624-639, June 2016. ISSN 0377-2217. doi: 10.1016/j.ejor.2015.11.022.

B. Ben Mabrouk, H. Hasni, and Z. Mahjoub. On a parallel genetic-tabu search based algorithm for solving the graph colouring problem. European Journal of Operational Research, 197(3):1192-1201, Sept. 2009. ISSN 0377-2217. doi: 10.1016/j.ejor.2008.03.050.

L. Benedicic, M. Stular, and P. Korosec. A GPU-based parallel-agent optimization approach for the service coverage problem in UMTS networks. Computing and Informatics, 33(5):1025-1046, 2014. ISSN 1335-9150.

A. Borisenko, P. Kegel, and S. Gorlatch. Optimal design of multi-product batch plants using a parallel branch-and-bound method. In Proceedings of the 11th International Conference on Parallel Computing Technologies, pages 417-430. Springer-Verlag Berlin, 2011.

A. Borisenko, M. Haidl, and S. Gorlatch. A GPU parallelization of branch-and-bound for multiproduct batch plants optimization. Journal of Supercomputing, 73(2):639-651, Feb. 2017. ISSN 0920-8542. doi: 10.1007/s11227-016-1784-x.

M. A. Boschetti, V. Maniezzo, and F. Strappaveccia. Using GPU computing for solving the two-dimensional guillotine cutting problem. INFORMS Journal on Computing, 28(3):540-552, SUM 2016. ISSN 1091-9856. doi: 10.1287/ijoc. 2016.0693.

A. Boukedjar, M. E. Lalami, and D. El-Baz. Parallel branch and bound on a CPU-GPU system. In Proceedings of the 20th Euromicro International Conference on Parallel, Distributed and Network-Based Processing (PDP), pages 392-398, 2012.

V. Boyer and D. El Baz. Recent advances on GPU computing in operations research. In Proceedings of the 27th International Conference on Parallel \& Distributed Processing Symposium, pages 1778-1787, 2013.

V. Boyer, D. El Baz, and M. Elkihel. Solving knapsack problems on GPU. Computers \& Operations Research, 39(1): 42-47, 2012.

D. Bozdağ, A. H. Gebremedhin, F. Manne, E. G. Boman, and U. V. Catalyurek. A framework for scalable greedy coloring on distributed-memory parallel computers. Journal of Parallel and Distributed Computing, 68(4):515-535, 2008. ISSN 0743-7315. doi: https://doi.org/10.1016/j.jpdc.2007.08.002. URL http://wWw.sciencedirect.com/ science/article/pii/S074373150700144X

W. Bożejko. Solving the flow shop problem by parallel programming. Journal of Parallel and Distributed Computing, 69(5):470-481, 2009.

W. Bożejko, J. Pempera, and C. Smutnicki. Parallel simulated annealing for the job shop scheduling problem. In Proceedings of the 9th International Conference on Computational Science, pages 631-640. Springer-Verlag Berlin, 2009.

W. Bozejko, J. Pempera, and C. Smutnicki. Parallel tabu search algorithm for the hybrid flow shop problem. Computers \& Industrial Engineering, 65(3):466-474, July 2013. ISSN 0360-8352.

W. Bożejko, M. Uchroński, and M. Wodecki. Parallel metaheuristics for the cyclic flow shop scheduling problem. Computers \& Industrial Engineering, 95:156-163, 2016.

W. Bozejko, A. Gnatowski, J. Pempera, and M. Wodecki. Parallel tabu search for the cyclic job shop scheduling problem. Computers \& Industrial Engineering, 113:512-524, Nov. 2017. ISSN 0360-8352.

A. R. Brodtkorb, T. R. Hagen, C. Schulz, and G. Hasle. Gpu computing in discrete optimization. part i: Introduction to the gpu. EURO journal on transportation and logistics, 2(1-2):129-157, 2013. 
L. Bukata, P. Sucha, and Z. Hanzalek. Solving the resource constrained project scheduling problem using the parallel tabu search designed for the CUDA platform. Journal of Parallel and Distributed Computing, 77:58-68, Mar. 2015. ISSN 0743-7315. doi: 10.1016/j.jpdc.2014.11.005.

Y. Caniou, D. Diaz, F. Richoux, P. Codognet, and S. Abreu. Performance analysis of parallel constraint-based local search. In Proceedings of the 17th ACM SIGPLAN Symposium on Principles and Practice of Parallel Programming, PPoPP '12, pages 337-338, New York, NY, USA, 2012. ISBN 978-1-4503-1160-1. doi: 10.1145/2145816.2145883. URL http://doi.acm.org/10.1145/2145816.2145883

E. Cantú-Paz. Theory of parallel genetic algorithms, chapter 18, pages 423-445. John Wiley \& Sons, Inc., Hoboken, New Jersey., 2005. ISBN 9780471739388.

E. Cantú-Paz. A survey of parallel genetic algorithms. Calculateurs Paralleles, Reseaux Et Systems Repartis, 10(2): 141-171, 1998.

B. Cao, J. Zhao, Z. Lv, and X. Liu. A distributed parallel cooperative coevolutionary multiobjective evolutionary algorithm for large-scale optimization. IEEE Transactions on Industrial Informatics, 13(4):2030-2038, Aug. 2017. ISSN 1551-3203. doi: 10.1109/TII.2017.2676000.

T. Carneiro, A. E. Muritiba, M. Negreiros, and G. A. L. de Campos. A new parallel schema for branch-and-bound algorithms using GPGPU. In Proceedings of the 23rd International Symposium on Computer Architecture and High Performance Computing, pages 41-47, 2011.

R. Carvajal, S. Ahmed, G. Nemhauser, K. Furman, V. Goel, and Y. Shao. Using diversification, communication and parallelism to solve mixed-integer linear programs. Operations Research Letters, 42(2):186-189, Mar. 2014. ISSN 0167-6377. doi: 10.1016/j.orl.2013.12.012.

S. Cauley, V. Balakrishnan, Y. C. Hu, and C.-k. Koh. A parallel branch-and-cut approach for detailed placement. ACM Transactions on Design Automation of Electronic Systems, 16(2), Mar. 2011. ISSN 1084-4309. doi: 10.1145/ 1929943.1929950.

J. M. Cecilia, J. M. Garcia, M. Ujaldon, A. Nisbet, and M. Amos. Parallelization strategies for ant colony optimisation on GPUs. In Proceedings of the 25th International Conference on Parallel \& Distributed Processing Symposium, pages 339-346, 2011. doi: 10.1109/ipdps.2011.170.

J. M. Cecilia, J. M. Garcia, A. Nisbet, M. Amos, and M. Ujaldon. Enhancing data parallelism for ant colony optimization on GPUs. Journal of Parallel and Distributed Computing, 73(1, SI):42-51, Jan. 2013. ISSN 0743-7315. doi: 10.1016/j.jpdc.2012.01.002.

I. Chakroun and N. Melab. Towards a heterogeneous and adaptive parallel branch-and-bound algorithm. Journal of Computer and System Sciences, 81(1, SI):72-84, Feb. 2015. ISSN 0022-0000. doi: 10.1016/j.jcss.2014.06.012.

I. Chakroun, N. Melab, M. Mezmaz, and D. Tuyttens. Combining multi-core and GPU computing for solving combinatorial optimization problems. Journal of Parallel and Distributed Computing, 73(12, SI):1563-1577, Dec. 2013a. ISSN 0743-7315. doi: 10.1016/j.jpdc.2013.07.023.

I. Chakroun, M. Mezmaz, N. Melab, and A. Bendjoudi. Reducing thread divergence in a GPU-accelerated branch-andbound algorithm. Concurrency and Computation-practice \& Experience, 25(8, SI):1121-1136, June 2013b. ISSN 1532-0626. doi: 10.1002/cpe.2931.

J. M. Chaves-Gonzalez, M. A. Vega-Rodriguez, J. A. Gomez-Pulido, and J. M. Sanchez-Perez. Optimizing a realistic large-scale frequency assignment problem using a new parallel evolutionary approach. Engineering Optimization, 43 (8):813-842, 2011. ISSN 0305-215X. doi: 10.1080/0305215X.2010.521241.

I. T. Christou and S. Vassilaras. A parallel hybrid greedy branch and bound scheme for the maximum distance-2 matching problem. Computers \& Operations Research, 40(10):2387-2397, Oct. 2013. ISSN 0305-0548. doi: 10.1016/j.cor.2013.04.009.

I. M. Coelho, P. L. A. Munhoz, L. S. Ochi, M. J. F. Souza, C. Bentes, and R. Farias. An integrated CPU-GPU heuristic inspired on variable neighbourhood search for the single vehicle routing problem with deliveries and selective pickups. International Journal of Production Research, 54(4):945-962, 2016. ISSN 0020-7543. doi: 10.1080/00207543.2015.1035811.

J.-F. Cordeau and M. Maischberger. A parallel iterated tabu search heuristic for vehicle routing problems. Computers \& Operations Research, 39(9):2033-2050, Sept. 2012. ISSN 0305-0548.

C. Cotta, E.-G. Talbi, and E. Alba. Parallel hybrid metaheuristics, chapter 15, pages 347-370. John Wiley \& Sons, Inc., Hoboken, New Jersey, 2005. ISBN 9780471739388.

T. Crainic and M. Toulouse. Learning and Intelligent Optimization, volume 5315 of Lecture Notes in Computer Science, chapter Explicit and emergent cooperation schemes for search algorithms, pages 95-109. Springer, Berlin, 2008. 
T. G. Crainic. Parallel solution methods for vehicle routing problems. In The Vehicle Routing Problem: Latest Advances and New Challenges, pages 171-198. Springer, Boston, MA, 2008.

T. G. Crainic. Parallel Meta-heuristic Search. In R. Martí, P. Panos, and M. G. Resende, editors, Handbook of Heuristics, pages 1-39. Springer, Cham, 2018.

T. G. Crainic. Parallel metaheuristics and cooperative search. In M. Gendreau and J.-Y. Potvin, editors, Handbook of Metaheuristics, pages 419-451. Springer, third edition, 2019.

T. G. Crainic and N. Hail. Parallel metaheuristics applications, chapter 1, pages 447-494. John Wiley \& Sons, Inc., Hoboken, New Jersey, 2005.

T. G. Crainic and M. Toulouse. Parallel strategies for meta-heuristics. In Handbook of metaheuristics, pages 475-513. Springer, Boston, MA, 2003.

T. G. Crainic and M. Toulouse. Parallel meta-heuristics. In M. Gendreau and J.-Y. Potvin, editors, Handbook of metaheuristics, pages 497-541. Springer, second edition, 2010.

T. G. Crainic, M. Gendreau, and J.-Y. Potvin. Parallel tabu search, chapter 13, pages 289-313. John Wiley \& Sons, Inc., Hoboken, New Jersey, 2005. ISBN 9780471739388.

T. G. Crainic, B. Le Cun, and C. Roucairol. Parallel combinatorial optimization, chapter Parallel Branch-and-Bound Algorithms, pages 1-28. John Wiley \& Sons, Inc., Hoboken, New Jersey, 2006. ISBN 9780470053928.

T. G. Crainic, T. Davidović, and D. Ramljak. Designing parallel meta-heuristic methods. In M. Despotovic-Zrakic, V. Milutinovic, and A. Belic, editors, Handbook of Research on High Performance and Cloud Computing in Scientific Research and Education, chapter 11, pages 260-280. IGI Global, Hershey, PA, 2014.

V.-D. Cung, S. L. Martins, C. C. Ribeiro, and C. Roucairol. Strategies for the parallel implementation of metaheuristics. In Essays and Surveys in Metaheuristics, pages 263-308. Springer, Boston, MA, 2002.

M. Czapinski. Parallel simulated annealing with genetic enhancement for flowshop problem with CSUM. Computers \& Industrial Engineering, 59(4):778-785, Nov. 2010. ISSN 0360-8352. doi: 10.1016/j.cie.2010.08.003.

M. Czapiński. An effective parallel multistart tabu search for quadratic assignment problem on CUDA platform. Journal of Parallel and Distributed Computing, 73(11):1461-1468, 2013.

M. Czapinski and S. Barnes. Tabu search with two approaches to parallel flowshop evaluation on CUDA platform. Journal of Parallel and Distributed Computing, 71(6, SI):802-811, June 2011. ISSN 0743-7315. doi: 10.1016/j.jpdc. 2011.02.006.

C. Dai, M. Toulouse, and B. P. C. Li. A multilevel cooperative tabu search algorithm for the covering design problem. The Journal of Combinatorial Mathematics and Combinatorial Computing, (68):35-65, 2009.

T. Davidović and T. G. Crainic. MPI parallelization of variable neighborhood search. Electronic Notes in Discrete Mathematics, 39:241-248, 2012.

T. Davidovic, D. Ramljak, M. Šelmic, and D. Teodorovic. Mpi parallelization of bee colony optimization. In Proc. 1st International Symposium \& 10th Balkan Conference on Operational Research, volume 2, pages 193-200, 2011.

K. Deep, S. Sharma, and M. Pant. Modified parallel particle swarm optimization for global optimization using message passing interface. In Proceedings of the 5th International Conference on Bio-Inspired Computing: Theories and Applications (BIC-TA), pages 1451-1458, 2010.

F. M. Defersha. A simulated annealing with multiple-search paths and parallel computation for a comprehensive flowshop scheduling problem. International Transactions in Operational Research, 22(4):669-691, July 2015. ISSN 0969-6016. doi: 10.1111/itor.12105.

F. M. Defersha and M. Chen. A parallel genetic algorithm for dynamic cell formation in cellular manufacturing systems. International Journal of Production Research, 46(22):6389-6413, 2008. ISSN 0020-7543. doi: 10.1080/ 00207540701441962.

F. M. Defersha and M. Chen. A parallel genetic algorithm for a flexible job-shop scheduling problem with sequence dependent setups. International Journal of Advanced Manufacturing Technology, 49(1-4):263-279, July 2010. ISSN 0268-3768. doi: 10.1007/s00170-009-2388-x.

F. M. Defersha and M. Chen. Mathematical model and parallel genetic algorithm for hybrid flexible flowshop lot streaming problem. International Journal of Advanced Manufacturing Technology, 62(1-4):249-265, Sept. 2012. ISSN 0268-3768. doi: 10.1007/s00170-011-3798-0.

A. Delevacq, P. Delisle, M. Gravel, and M. Krajecki. Parallel ant colony optimization on graphics processing units. Journal of Parallel and Distributed Computing, 73(1, SI):52-61, Jan. 2013. ISSN 0743-7315. doi: 10.1016/j.jpdc. 2012.01.003. 
B. Derbel, J. Humeauc, A. Liefooghe, and S. Verel. Distributed localized bi-objective search. European Journal of Operational Research, 239(3):731-743, Dec. 2014. ISSN 0377-2217. doi: 10.1016/j.ejor.2014.05.040.

B. H. Dias, M. A. Tomim, A. L. Marques Marcato, T. P. Ramos, R. B. S. Brandi, I. C. Da Silva Junior, and J. A. Passos Filho. Parallel computing applied to the stochastic dynamic programming for long term operation planning of hydrothermal power systems. European Journal of Operational Research, 229(1):212-222, Aug. 2013. ISSN 0377-2217.

J. Diaz, C. Munoz-Caro, and A. Nino. A survey of parallel programming models and tools in the multi and many-core era. IEEE Transactions on parallel and distributed systems, 23(8):1369-1386, 2012.

F. J. Diego, E. M. Gómez, M. Ortega-Mier, and Á. García-Sánchez. Parallel CUDA architecture for solving de VRP with ACO. In Industrial Engineering: Innovative Networks, pages 385-393. Springer, Boston, MA, 2012.

K. Ding, S. Zheng, and Y. Tan. A GPU-based parallel fireworks algorithm for optimization. In Proceedings of the 15th annual conference on Genetic and evolutionary computation, pages 9-16, 2013.

A. Djerrah, B. Le Cun, V.-D. Cung, and C. Roucairol. Bob++: Framework for solving optimization problems with branch-and-bound methods. In Proceedings of the 15th IEEE International Conference on High Performance Distributed Computing, pages 369-370, 2006.

F. Dobrian, A. Gebremedhin, M. Halappanavar, A. Pothen, et al. Distributed-memory parallel algorithms for matching and coloring. In Proceedings of the 26th International Parallel and Distributed Processing Symposium Workshops \& PhD Forum, pages 1971-1980, 2011.

G. Dongdong, G. Guanghong, H. Liang, and L. Ni. Application of multi-core parallel ant colony optimization in target assignment problem. In Proceedings of the 2010 International Conference on Computer Application and System Modeling (ICCASM), volume 3, pages V3-514, 2010.

M. Dorigo and T. Stützle. Ant colony optimization. MIT Press, 2004.

B. Dorronsoro, G. Danoy, A. J. Nebro, and P. Bouvry. Achieving super-linear performance in parallel multi-objective evolutionary algorithms by means of cooperative coevolution. Computers \& Operations Research, 40(6, SI): 1552-1563, June 2013. ISSN 0305-0548. doi: 10.1016/j.cor.2011.11.014.

J. Eckstein, W. E. Hart, and C. A. Phillips. PEBBL: an object-oriented framework for scalable parallel branch and bound. Mathematical Programming Computation, 7(4):429-469, 2015.

M. Eskandarpour, S. H. Zegordi, and E. Nikbakhsh. A parallel variable neighborhood search for the multi-objective sustainable post-sales network design problem. International Journal of Production Economics, 145(1):117-131, Sept. 2013. ISSN 0925-5273. doi: 10.1016/j.ijpe.2012.10.013.

F. Fabris and R. A. Krohling. A co-evolutionary differential evolution algorithm for solving min-max optimization problems implemented on GPU using c-CUDA. Expert Systems with Applications, 39(12):10324-10333, Sept. 2012. ISSN 0957-4174. doi: 10.1016/j.eswa.2011.10.015.

A. M. Ferreiro, J. A. Garcia, J. G. Lopez-Salas, and C. Vazquez. An efficient implementation of parallel simulated annealing algorithm in GPUs. Journal of Global Optimization, 57(3, SI):863-890, Nov. 2013. ISSN 0925-5001. doi: 10.1007/s10898-012-9979-z.

J. R. Figueira, A. Liefooghe, E. G. Talbi, and A. P. Wierzbicki. A parallel multiple reference point approach for multi-objective optimization. European Journal of Operational Research, 205(2):390-400, Sept. 2010. ISSN 0377-2217. doi: 10.1016/j.ejor.2009.12.027.

N. Fujimoto and S. Tsutsui. A highly-parallel TSP solver for a GPU computing platform. In Proceedings of the 7th. International Conference on Numerical Methods and Applications, pages 264-271. Springer-Verlag Berlin, 2011.

F. Galea and B. Le Cun. A parallel exact solver for the three-index quadratic assignment problem. In Proceedings of the 2011 IEEE International Symposium on Parallel and Distributed Processing Workshops and PhD Forum, pages 1940-1949, 2011.

J. Gao, G. He, and Y. Wang. A new parallel genetic algorithm for solving multiobjective scheduling problems subjected to special process constraint. International Journal of Advanced Manufacturing Technology, 43(1-2):151-160, July 2009. ISSN 0268-3768. doi: 10.1007/s00170-008-1683-2.

M. Gendreau, J.-Y. Potvin, et al., editors. Handbook of metaheuristics, volume 146 of International Series in Operations Research \& Management Science. Springer, third edition, 2010.

M. Gendreau, J.-Y. Potvin, et al., editors. Handbook of metaheuristics, volume 146 of International Series in Operations Research \& Management Science. Springer, 2019. 
B. Gendron and T. G. Crainic. Parallel branch-and-branch algorithms: survey and synthesis. Operations Research, 42 (6):1042-1066, 1994.

T. E. Gerasch and P. Y. Wang. A survey of parallel algorithms for one-dimensional integer knapsack problems. INFOR: Information Systems and Operational Research, 32(3):163-186, 1994.

J. Gmys, M. Mezmaz, N. Melab, and D. Tuyttens. A GPU-based branch-and-bound algorithm using integer-vectormatrix data structure. Parallel Computing, 59(SI):119-139, Nov. 2016. ISSN 0167-8191. doi: 10.1016/j.parco.2016. 01.008 .

J. Gmys, M. Mezmaz, N. Melab, and D. Tuyttens. Ivm-based parallel branch-and-bound using hierarchical work stealing on multi-GPU systems. Concurrency and Computation-practice \& Experience, 29(9, SI), May 2017. ISSN 1532-0626. doi: 10.1002/cpe.4019.

F. C. Gomes, C. N. Meneses, P. M. Pardalos, and G. V. R. Viana. A parallel multistart algorithm for the closest string problem. Computers \& Operations Research, 35(11):3636-3643, Nov. 2008. ISSN 0305-0548. doi: 10.1016/j.cor.2007.04.002.

C. Groer, B. Golden, and E. Wasil. A parallel algorithm for the vehicle routing problem. INFORMS Journal on Computing, 23(2):315-330, SPR 2011. ISSN 1091-9856. doi: 10.1287/ijoc.1100.0402.

A. Hadian, S. Shahrivari, and B. Minaei-Bidgoli. Fine-grained parallel ant colony system for shared-memory architectures. International Journal of Computer Applications, 53(8), 2012.

J. He, D. Chang, W. Mi, and W. Yan. A hybrid parallel genetic algorithm for yard crane scheduling. Transportation Research Part E-logistics and Transportation Review, 46(1):136-155, Jan. 2010. ISSN 1366-5545. doi: 10.1016/j. tre.2009.07.002.

V. C. Hemmelmayr. Sequential and parallel large neighborhood search algorithms for the periodic location routing problem. European Journal of Operational Research, 243(1):52-60, May 2015. ISSN 0377-2217. doi: 10.1016/j. ejor.2014.11.024.

J. F. Herrera, L. G. Casado, E. M. Hendrix, R. Paulavicius, and J. Ilinskas. Dynamic and hierarchical load-balancing techniques applied to parallel branch-and-bound methods. In Proceedings of the 8th International Conference on P2P, Parallel, Grid, Cloud and Internet Computing (3PGCIC), pages 497-502, 2013.

J. F. R. Herrera, J. M. G. Salmeron, E. M. T. Hendrix, R. Asenjo, and L. G. Casado. On parallel branch and bound frameworks for global optimization. Journal of Global Optimization, 69(3):547-560, Nov. 2017. ISSN 0925-5001.

M. Hifi, S. Negre, T. Saadi, S. Saleh, and L. Wu. A parallel large neighborhood search-based heuristic for the disjunctively constrained knapsack problem. In Proceedings of the 2014 IEEE International Parallel \& Distributed Processing Symposium Workshops, pages 1547-1551, 2014.

J. Homberger. A parallel genetic algorithm for the multilevel unconstrained lot-sizing problem. INFORMS Journal on Computing, 20(1):124-132, WIN 2008. ISSN 1091-9856. doi: 10.1287/ijoc.1070.0224.

L. Hong, L. Zhong-hua, and C. Xue-bin. Parallel computing for dynamic asset allocation based on the stochastic programming. In Proceedings of the 2010 WASE International Conference on Information Engineering (ICIE), volume 2, pages 172-176, 2010.

N. Hou, F. He, Y. Zhou, and H. Ai. A GPU-based tabu search for very large hardware/software partitioning with limited resource usage. Journal of Advanced Mechanical Design Systems and Manufacturing, 11(5), 2017. ISSN 1881-3054. doi: 10.1299/jamdsm.2017jamdsm0060.

C.-S. Huang, Y.-C. Huang, and P.-J. Lai. Modified genetic algorithms for solving fuzzy flow shop scheduling problems and their implementation with CUDA. Expert Systems with Applications, 39(5):4999-5005, Apr. 2012. ISSN 0957-4174. doi: 10.1016/j.eswa.2011.10.013.

J. Huebner, M. Schmidt, and M. C. Steinbach. A distributed interior-point KKT solver for multistage stochastic optimization. INFORMS Journal on Computing, 29(4):612-630, FAL 2017. ISSN 1091-9856. doi: 10.1287/ijoc. 2017.0748.

Y. Hung and W. Wang. Accelerating parallel particle swarm optimization via GPU. Optimization Methods \& Software, 27(1):33-51, 2012. ISSN 1055-6788. doi: 10.1080/10556788.2010.509435.

S. Ibri, H. Drias, and M. Nourelfath. A parallel hybrid ant-tabu algorithm for integrated emergency vehicle dispatching and covering problem. International Journal of Innovative Computing and Applications, 2(4):226-236, 2010.

INRIA. Paradiseo - A Software Framewok for Metaheuristics. http://paradiseo.gforge.inria.fr, n.d.

M. A. Ismail, S. H. Mirza, and T. Altaf. A parallel and concurrent implementation of Lin-Kernighan heuristic (LKH-2) for solving traveling salesman problem for multi-core processors using SPC 3 programming model. International Journal of Advanced Computer Science and Applications, 2011. 
M. M. Ismail, O. Abd El-Raoof, and W. F. Abd El-Wahed. A parallel branch and bound algorithm for solving large scale integer programming problems. Applied Mathematics \& Information Sciences, 8(4):1691-1698, July 2014. ISSN 2325-0399. doi: 10.12785/amis/080425.

D. Izzo, M. Rucinski, and C. Ampatzis. Parallel global optimisation meta-heuristics using an asynchronous island-model. In 2009 IEEE Congress on Evolutionary Computation, pages 2301-2308. IEEE, 2009.

T. James, C. Rego, and F. Glover. A cooperative parallel tabu search algorithm for the quadratic assignment problem. European Journal of Operational Research, 195(3):810-826, June 2009. ISSN 0377-2217. doi: 10.1016/j.ejor.2007. 06.061 .

A. Janiak, W. Janiak, and M. Lichtenstein. Tabu search on GPU. Journal of Universal Computer Science, 14(14): 2416-2427, 2008. ISSN 0948-695X.

S. Janson, D. Merkle, and M. Middendorf. Parallel ant colony algorithms, chapter 3, pages 171-201. John Wiley \& Sons, Inc., Hoboken, New Jersey, 2005.

J. Jin, T. G. Crainic, and A. Løkketangen. A guided cooperative parallel tabu search for the capacitated vehicle routing problem. In Proceedings of NIK 2011, pages 49-60, 2011.

J. Jin, T. G. Crainic, and A. Lokketangen. A parallel multi-neighborhood cooperative tabu search for capacitated vehicle routing problems. European Journal of Operational Research, 222(3):441-451, Nov. 2012. ISSN 0377-2217. doi: 10.1016/j.ejor.2012.05.025.

J. Jin, T. G. Crainic, and A. Lokketangen. A cooperative parallel metaheuristic for the capacitated vehicle routing problem. Computers \& Operations Research, 44:33-41, Apr. 2014. ISSN 0305-0548. doi: 10.1016/j.cor.2013.10.004.

A. A. Juan, J. Faulin, J. Jorba, J. Caceres, and J. Manuel Marques. Using parallel \& distributed computing for real-time solving of vehicle routing problems with stochastic demands. Annals of Operations Research, 207(1):43-65, Aug. 2013. ISSN 0254-5330. doi: 10.1007/s10479-011-0918-z.

S. Kang, S.-S. Kim, J. Won, and Y.-M. Kang. GPU-based parallel genetic approach to large-scale travelling salesman problem. Journal of Supercomputing, 72(11):4399-4414, Nov. 2016. ISSN 0920-8542. doi: 10.1007/s11227-016-1748-1.

L. P. Kerkhove and M. Vanhoucke. A parallel multi-objective scatter search for optimising incentive contract design in projects. European Journal of Operational Research, 261(3):1066-1084, Sept. 2017. ISSN 0377-2217. doi: 10.1016/j.ejor.2017.02.043.

D. S. Knysh and V. M. Kureichik. Parallel genetic algorithms: a survey and problem state of the art. Journal of Computer and Systems Sciences International, 49(4):579-589, Aug. 2010. ISSN 1064-2307.

U. Koc and S. Mehrotra. Generation of feasible integer solutions on a massively parallel computer using the feasibility pump. Operations Research Letters, 45(6):652-658, Nov. 2017. ISSN 0167-6377. doi: 10.1016/j.orl.2017.10.003.

G. Kollias, M. Sathe, O. Schenk, and A. Grama. Fast parallel algorithms for graph similarity and matching. Journal of Parallel and Distributed Computing, 74(5):2400-2410, May 2014. ISSN 0743-7315. doi: https://doi.org/10.1016/j. jpdc.2013.12.010. URL http://www.sciencedirect.com/science/article/pii/S0743731513002529.

M.-Y. Ku, M. H. Hu, and M.-J. Wang. Simulated annealing based parallel genetic algorithm for facility layout problem. International Journal of Production Research, 49(6):1801-1812, 2011. ISSN 0020-7543. doi: 10.1080/ 00207541003645789.

S. Kumar, A. Misra, and R. S. Tomar. A modified parallel approach to single source shortest path problem for massively dense graphs using CUDA. In Proceedings of the 2nd International Conference on Computer and Communication Technology (ICCCT), pages 635-639, 2011.

G. A. Laguna-Sanchez, M. Olguin-Carbajal, N. Cruz-Cortes, R. Barron-Fernandez, and J. A. Alvarez-Cedillo. Comparative study of parallel variants for a particle swarm optimization algorithm implemented on a multithreading GPU. Journal of Applied Research and Technology, 7(3):292-309, Dec. 2009. ISSN 1665-6423.

N. Lahrichi, T. G. Crainic, M. Gendreau, W. Rei, G. C. Crişan, and T. Vidal. An integrative cooperative search framework for multi-decision-attribute combinatorial optimization: Application to the mdpvrp. European Journal of Operational Research, 246(2):400-412, 2015.

A. Lančinskas and J. Żilinskas. Solution of multi-objective competitive facility location problems using parallel NSGA-II on large scale computing systems. In Proceedings of the 11th International Conference on Applied Parallel Computing, pages 422-433. Springer-Verlag Berlin, 2012.

A. Lančinskas and J. Žilinskas. Parallel multi-objective memetic algorithm for competitive facility location. In Proceedings of the 10th International Conference on Parallel Processing and Applied Mathematics, pages 354-363. Springer-Verlag Berlin, 2013. 
A. Lancinskas, P. Martinez Ortigosa, and J. Zilinskas. Parallel optimization algorithm for competitive facility location. Mathematical Modelling and Analysis, 20(5):619-640, Sept. 2015. ISSN 1392-6292. doi: 10.3846/13926292.2015. 1088903.

M. Lazarova and P. Borovska. Comparison of parallel metaheuristics for solving the TSP. In Proceedings of the 9th International Conference on Computer Systems and Technologies and Workshop for PhD Students in Computing, page 17, 2008.

D. Lei and X. Guo. A parallel neighborhood search for order acceptance and scheduling in flow shop environment. International Journal of Production Economics, 165:12-18, July 2015. ISSN 0925-5273. doi: 10.1016/j.ijpe.2015. 03.013.

C.-C. Li, C.-H. Lin, and J.-C. Liu. Parallel genetic algorithms on the graphics processing units using island model and simulated annealing. Advances in Mechanical Engineering, 9(7), July 2017. ISSN 1687-8140. doi: 10.1177/ 1687814017707413.

K. Li, J. Liu, L. Wan, S. Yin, and K. Li. A cost-optimal parallel algorithm for the 0-1 knapsack problem and its performance on multicore CPU and GPU implementations. Parallel Computing, 43:27-42, Mar. 2015. ISSN 0167-8191. doi: 10.1016/j.parco.2015.01.004.

S. Limmer and D. Fey. Comparison of common parallel architectures for the execution of the island model and the global parallelization of evolutionary algorithms. Concurrency and Computation: Practice and Experience, 29(9), 2017.

C. Ling, S. Hai-Ying, and W. Shu. A parallel ant colony algorithm on massively parallel processors and its convergence analysis for the travelling salesman problem. Information Sciences, 199:31-42, Sept. 2012. ISSN 0020-0255. doi: 10.1016/j.ins.2012.02.055.

K.-H. Liu and J.-J. Kao. Parallelised branch-and-bound algorithm for raster-based landfill siting. Civil Engineering and Environmental Systems, 30(1):15-25, Mar. 2013. ISSN 1028-6608. doi: 10.1080/10286608.2012.709504.

Y. Y. Liu and S. Wang. A scalable parallel genetic algorithm for the generalized assignment problem. Parallel Computing, 46:98-119, July 2015. ISSN 0167-8191. doi: 10.1016/j.parco.2014.04.008.

Y. Y. Liu, W. K. T. Cho, and S. Wang. Pear: a massively parallel evolutionary computation approach for political redistricting optimization and analysis. Swarm and Evolutionary Computation, 30:78-92, Oct. 2016. ISSN 2210-6502. doi: 10.1016/j.swevo.2016.04.004.

F. A. Lootsma and K. M. Ragsdell. State-of-the-art in parallel nonlinear optimization. Parallel Computing, 6(2): 133-155, Feb. 1988. ISSN 0167-8191.

Z. Lou and J. Reinitz. Parallel simulated annealing using an adaptive resampling interval. Parallel Computing, 53: 23-31, Apr. 2016. ISSN 0167-8191.

F. G. López, M. G. Torres, B. M. Batista, J. A. M. Perez, and J. M. M. Vega. Parallel scatter search, chapter 10, pages 223-246. John Wiley \& Sons, Inc., Hoboken, New Jersey., 2005. ISBN 9780471739388.

H. Lu, J. Liu, R. Niu, and Z. Zhu. Fitness distance analysis for parallel genetic algorithm in the test task scheduling problem. Soft Computing, 18(12):2385-2396, Dec. 2014. ISSN 1432-7643.

M. Lubin, C. G. Petra, and M. Anitescu. The parallel solution of dense saddle-point linear systems arising in stochastic programming. Optimization Methods and Software, 27(4-5):845-864, 2012.

M. Lubin, K. Martin, C. G. Petra, and B. Sandikci. On parallelizing dual decomposition in stochastic integer programming. Operations Research Letters, 41(3):252-258, May 2013. ISSN 0167-6377.

M. Lucka, I. Melichercik, and L. Halada. Application of multistage stochastic programs solved in parallel in portfolio management. Parallel Computing, 34(6-8):469-485, 2008.

F. Luna, E. Alba, and A. J. Nebro. Parallel heterogeneous metaheuristics, chapter 17, pages 395-422. John Wiley \& Sons, Inc., Hoboken, New Jersey, 2005. ISBN 9780471739388.

G.-H. Luo, S.-K. Huang, Y.-S. Chang, and S.-M. Yuan. A parallel bees algorithm implementation on GPU. Journal of Systems Architecture, 60(3):271-279, 2014.

J. Luo, L. J. Hong, B. L. Nelson, and Y. Wu. Fully sequential procedures for large-scale ranking-and-selection problems in parallel computing environments. Operations Research, 63(5):1177-1194, 2015.

G. Luque, E. Alba, and B. Dorronsoro. Parallel genetic algorithms, chapter 5, pages 105-125. John Wiley \& Sons, Inc., Hoboken, New Jersey, 2005. ISBN 9780471739388.

M. Maischberger and J.-F. Cordeau. Solving variants of the vehicle routing problem with a simple parallel iterated tabu search. In Network optimization, pages 395-400. Springer-Verlag Berlin, 2011. 
S. Maleki, M. Musuvathi, and T. Mytkowicz. Efficient parallelization using rank convergence in dynamic programming algorithms. Communications of the ACM, 59(10):85-92, Oct. 2016. ISSN 0001-0782. doi: 10.1145/2983553.

S. L. Martins and C. C. Ribeiro. Metaheuristics and applications to optimization problems in telecommunications. In Handbook of Optimization in Telecommunications, pages 103-128. Springer, Boston, MA, 2006.

R. Massobrio, J. Toutouh, S. Nesmachnow, and E. Alba. Infrastructure deployment in vehicular communication networks using a parallel multiobjective evolutionary algorithm. International Journal of Intelligent Systems, 32(8): 801-829, Aug. 2017. ISSN 0884-8173. doi: 10.1002/int.21890.

C. McCreesh and P. Prosser. A parallel branch and bound algorithm for the maximum labelled clique problem. Optimization Letters, 9(5):949-960, June 2015. ISSN 1862-4472. doi: 10.1007/s11590-014-0837-4.

N. Melab, E.-g. Talbi, S. Cahon, E. Alba, and G. Luque. Parallel metaheuristics: algorithms and frameworks, chapter 6, pages 149-161. John Wiley \& Sons, Inc., Hoboken, New Jersey, 2006. ISBN 9780470053928.

N. Melab, T. Luong, K. Boufaras, and E.-G. Talbi. Towards paradiseo-mo-gpu: a framework for gpu-based local search metaheuristics. In International Work-Conference on Artificial Neural Networks, pages 401-408. Springer, 2011.

B. Menendez, E. G. Pardo, J. Sanchez-Oro, and A. Duarte. Parallel variable neighborhood search for the min-max order batching problem. International Transactions in Operational Research, 24(3):635-662, May 2017. ISSN 0969-6016. doi: 10.1111/itor.12309.

M. Mezmaz, N. Melab, Y. Kessaci, Y. C. Lee, E. G. Talbi, A. Y. Zomaya, and D. Tuyttens. A parallel bi-objective hybrid metaheuristic for energy-aware scheduling for cloud computing systems. Journal of Parallel and Distributed Computing, 71(11):1497-1508, Nov. 2011. ISSN 0743-7315. doi: 10.1016/j.jpdc.2011.04.007.

M. Mezmaz, R. Leroy, N. Melab, and D. Tuyttens. A multi-core parallel branch-and-bound algorithm using factorial number system. In Proceedings of the 28th International Symposium on Parallel and Distributed Processing, pages 1203-1212, 2014. doi: 10.1109/ipdps.2014.124.

D. Mu, C. Wang, F. Zhao, and J. W. Sutherland. Solving vehicle routing problem with simultaneous pickup and delivery using parallel simulated annealing algorithm. International Journal of Shipping and Transport Logistics, 8 (1):81-106, 2016. ISSN 1756-6517. doi: 10.1504/IJSTL.2016.073323.

A. Munawar, M. Wahib, M. Munetomo, and K. Akama. Hybrid of genetic algorithm and local search to solve MAX-SAT problem using nvidia CUDA framework. Genetic Programming and Evolvable Machines, 10(4):391-415, Dec. 2009. ISSN 1389-2576. doi: 10.1007/s10710-009-9091-4.

L. Mussi, F. Daolio, and S. Cagnoni. Evaluation of parallel particle swarm optimization algorithms within the CUDA architecture. Information Sciences, 181(20):4642-4657, Oct. 2011. ISSN 0020-0255. doi: 10.1016/j.ins.2010.08.045.

A. J. Nebro and J. J. Durillo. A study of the parallelization of the multi-objective metaheuristic MOEA/D. In Proceedings of the 11th International Conference on Learning and Intelligent Optimization, pages 303-317. Springer-Verlag Berlin, 2010.

A. J. Nebro, F. Luna, E.-g. Talbi, and E. Alba. Parallel multiobjective optimization, chapter 16, pages 371-394. John Wiley \& Sons, Inc., Hoboken, New Jersey., 2005. ISBN 9780471739388.

S. Nesmachnow, H. Cancela, E. Alba, and F. Chicano. Parallel metaheuristics in telecommunications, chapter 20, pages 495-515. John Wiley \& Sons, Inc., Hoboken, New Jersey, 2005. ISBN 9780471739388.

S. Nesmachnow, H. Cancela, and E. Alba. A parallel micro evolutionary algorithm for heterogeneous computing and grid scheduling. Applied Soft Computing, 12(2):626-639, Feb. 2012. ISSN 1568-4946.

R. Nowotniak and J. Kucharski. GPU-based massively parallel implementation of metaheuristic algorithms. Automatyka/Akademia Górniczo-Hutnicza im. Stanisława Staszica w Krakowie, 15:595-611, 2011.

V. Nwana and Mitra. Parallel mixed integer programming: a status review. Technical report, Department of Mathematical Sciences, Brunel University, 2000.

J. Olensek, T. Tuma, J. Puhan, and A. Burmen. A new asynchronous parallel global optimization method based on simulated annealing and differential evolution. Applied Soft Computing, 11(1):1481-1489, Jan. 2011. ISSN 1568-4946. doi: 10.1016/j.asoc.2010.04.019.

S. G. Ozden, A. E. Smith, and K. R. Gue. Solving large batches of traveling salesman problems with parallel and distributed computing. Computers \& Operations Research, 85:87-96, Sept. 2017. ISSN 0305-0548.

A. Pages-Bernaus, G. Perez-Valdes, and A. Tomasgard. A parallelised distributed implementation of a branch and fix coordination algorithm. European Journal of Operational Research, 244(1):77-85, July 2015. ISSN 0377-2217. doi: 10.1016/j.ejor.2015.01.004. 
P. M. Pardalos, L. Pitsoulis, T. Mavridou, and M. G. C. Resende. Parallel search for combinatorial optimization: genetic algorithms, simulated annealing, tabu search and GRASP. In Proceedings of the 2nd International Workshop on Parallel Algorithms for Irregularly Structured Problems, pages 317-331, 1995.

C. Patvardhan, S. Bansal, and A. Srivastav. Parallel improved quantum inspired evolutionary algorithm to solve large size quadratic knapsack problems. Swarm and Evolutionary Computation, 26:175-190, Feb. 2016. ISSN 2210-6502. doi: 10.1016/j.swevo.2015.09.005.

R. Paulavičius and J. Žilinskas. Parallel branch and bound algorithm with combination of lipschitz bounds over multidimensional simplices for multicore computers. In Parallel Scientific Computing and Optimization, pages 93-102. Springer, Boston, MA, 2009.

R. Paulavicius, J. Zilinskas, and A. Grothey. Parallel branch and bound for global optimization with combination of lipschitz bounds. Optimization Methods \& Software, 26(3):487-498, 2011. ISSN 1055-6788. doi: 10.1080/ 10556788.2010.551537.

M. Pedemonte, S. Nesmachnow, and H. Cancela. A survey on parallel ant colony optimization. Applied Soft Computing, 11(8):5181-5197, 2011.

D. M. Pedroso, M. R. Bonyadi, and M. Gallagher. Parallel evolutionary algorithm for single and multi-objective optimisation: differential evolution and constraints handling. Applied Soft Computing, 61:995-1012, Dec. 2017. ISSN 1568-4946. doi: 10.1016/j.asoc.2017.09.006.

M. Polacek, S. Benkner, K. F. Doerner, and R. F. Hartl. A cooperative and adaptive variable neighborhood search for the multi depot vehicle routing problem with time windows. Business Research, 1(2):207-218, 2008.

O. Polat. A parallel variable neighborhood search for the vehicle routing problem with divisible deliveries and pickups. Computers \& Operations Research, 85:71-86, Sept. 2017. ISSN 0305-0548. doi: 10.1016/j.cor.2017.03.009.

J. L. Ponz-Tienda, A. Salcedo-Bernal, and E. Pellicer. A parallel branch and bound algorithm for the resource leveling problem with minimal lags. Computer-aided Civil and Infrastructure Engineering, 32(6):474-498, June 2017. ISSN 1093-9687. doi: 10.1111/mice.12233.

M. A. Posypkin and I. K. Sigal. A combined parallel algorithm for solving the knapsack problem. Journal of Computer and Systems Sciences International, 47(4):543-551, Aug. 2008. ISSN 1064-2307. doi: 10.1134/S1064230708040072.

J. A. M. Pérez, P. Hansen, and N. Mladenović. Parallel variable neighborhood search, chapter 11, pages 247-266. John Wiley \& Sons, Inc., Hoboken, New Jersey, 2005. ISBN 9780471739388.

J. Qu, X. Liu, M. Sun, and F. Qi. GPU-based parallel particle swarm optimization methods for graph drawing. Discrete Dynamics in Nature and Society, 2017. ISSN 1026-0226. doi: 10.1155/2017/2013673.

Z. Quan and L. Wu. Design and evaluation of a parallel neighbor algorithm for the disjunctively constrained knapsack problem. Concurrency and Computation-practice \& Experience, 29(20, SI), Oct. 2017. ISSN 1532-0626. doi: $10.1002 /$ cpe.3848.

M. Randall and A. Lewis. A parallel implementation of ant colony optimization. Journal of Parallel and Distributed Computing, 62(9):1421-1432, 2002.

H. Rashid, C. Novoa, and A. Qasem. An evaluation of parallel knapsack algorithms on multicore architectures. In Proceedings of the 2010 International Conference on Scientific Computing, pages 230-235, 2010.

M. G. Ravetti, C. Riveros, A. Mendes, M. G. C. Resende, and P. M. Pardalos. Parallel hybrid heuristics for the permutation flow shop problem. Annals of Operations Research, 199(1):269-284, Oct. 2012. ISSN 0254-5330. doi: 10.1007/s10479-011-1056-3.

J. L. Redondo, J. Fernandez, I. Garcia, and P. M. Ortigosa. Parallel algorithms for continuous competitive location problems. Optimisation Methods \& Software, 23(5):779-791, 2008.

J. L. Redondo, I. Garcia, and P. M. Ortigosa. Parallel evolutionary algorithms based on shared memory programming approaches. Journal of Supercomputing, 58(2, SI):270-279, Nov. 2011. ISSN 0920-8542. doi: 10.1007/s11227-009-0374-6.

J. L. Redondo, A. Marin, and P. M. Ortigosa. A parallelized lagrangean relaxation approach for the discrete ordered median problem. Annals of Operations Research, 246(1-2):253-272, Nov. 2016. ISSN 0254-5330. doi: 10.1007/ s10479-014-1744-X.

M. G. C. Resende and C. C. Ribeiro. Parallel greedy randomized adaptive search procedures, chapter 14, pages 315-346. John Wiley \& Sons, Inc., Hoboken, New Jersey., 2005. ISBN 9780471739388.

V. Roberge, M. Tarbouchi, and G. Labonte. Comparison of parallel genetic algorithm and particle swarm optimization for real-time UAV path planning. IEEE Transactions on Industrial Informatics, 9(1):132-141, Feb. 2013. ISSN 1551-3203. doi: 10.1109/TII.2012.2198665. 
M. Rossbory and W. Reisner. Parallelization of algorithms for linear discrete optimization using paraphrase. In Proceedings of the 24th International Workshop on Database and Expert Systems Applications (DEXA), pages 241-245, 2013.

R. Rudek. Exact and parallel metaheuristic algorithms for the single processor total weighted completion time scheduling problem with the sum-of-processing-time based models. Computers \& Operations Research, 46:91-101, June 2014. ISSN 0305-0548. doi: 10.1016/j.cor.2014.01.008.

G. Rudolph. Parallel evolution strategies, chapter 7, pages 155-169. John Wiley \& Sons, Inc., Hoboken, New Jersey, 2005. ISBN 9780471739388.

S. Sancı and V. İşler. A parallel algorithm for UAV flight route planning on GPU. International Journal of Parallel Programming, 39(6):809-837, 2011.

J. Sanjuan-Estrada, L. G. Casado, and I. García. Adaptive parallel interval global optimization algorithms based on their performance for non-dedicated multicore architectures. In Proceedings of the 19th Euromicro International Conference on Parallel, Distributed and Network-Based Processing (PDP), pages 252-256, 2011.

L. Santos, D. Madeira, E. Clua, S. Martins, and A. Plastino. A parallel GRASP resolution for a GPU architecture. In Proceedings of the 7th International Conference on Metaheuristics and Nature Inspired Computing, page META10, 2010.

M. Sathe, O. Schenk, and H. Burkhart. An auction-based weighted matching implementation on massively parallel architectures. Parallel Computing, 38(12):595-614, 2012.

K. Scheerlinck, H. Vernieuwe, and B. De Baets. Zadeh's extension principle for continuous functions of non-interactive variables: a parallel optimization approach. IEEE Transactions on Fuzzy Systems, 20(1):96-108, Feb. 2012. ISSN 1063-6706. doi: 10.1109/TFUZZ.2011.2168406.

C. Schulz, G. Hasle, A. R. Brodtkorb, and T. R. Hagen. GPU computing in discrete optimization: Part ii: Survey focused on routing problems. EURO Journal on Transportation and Logistics, 2:159-186, 2013.

O. V. Shylo, T. Middelkoop, and P. M. Pardalos. Restart strategies in optimization: parallel and serial cases. Parallel Computing, 37(1):60-68, Jan. 2011. ISSN 0167-8191. doi: 10.1016/j.parco.2010.08.004.

J. M. N. Silva, C. Boeres, L. M. A. Drummond, and A. A. Pessoa. Memory aware load balance strategy on a parallel branch-and-bound application. Concurrency and Computation-practice \& Experience, 27(5):1122-1144, Apr. 2015. ISSN 1532-0626. doi: 10.1002/cpe.3276.

R. Skinderowicz. The GPU-based parallel ant colony system. Journal of Parallel and Distributed Computing, 98: 48-60, Dec. 2016. ISSN 0743-7315. doi: 10.1016/j.jpdc.2016.04.014.

P. Stanojevic, M. Maric, and Z. Stanimirovic. A hybridization of an evolutionary algorithm and a parallel branch and bound for solving the capacitated single allocation hub location problem. Applied Soft Computing, 33:24-36, Aug. 2015. ISSN 1568-4946. doi: 10.1016/j.asoc.2015.04.018.

A. Stivala, P. J. Stuckey, M. Garcia De La Banda, M. Hermenegildo, and A. Wirth. Lock-free parallel dynamic programming. Journal of Parallel and Distributed Computing, 70(8):839-848, Aug. 2010. ISSN 0743-7315. doi: 10.1016/j.jpdc.2010.01.004.

M. Subotic, M. Tuba, and N. Stanarevic. Different approaches in parallelization of the artificial bee colony algorithm. International Journal of mathematical models and methods in applied sciences, 5(4):755-762, 2011.

A. Subramanian, L. M. A. Drummond, C. Bentes, L. S. Ochi, and R. Farias. A parallel heuristic for the vehicle routing problem with simultaneous pickup and delivery. Computers \& Operations Research, 37(11, SI):1899-1911, Nov. 2010. ISSN 0305-0548. doi: 10.1016/j.cor.2009.10.011.

E.-G. Talbi, editor. Parallel combinatorial optimization. John Wiley \& Sons, 2006.

E.-G. Talbi. Metaheuristics: from design to implementation. John Wiley \& Sons, 2009.

G. Tan, N. Sun, and G. R. Gao. Improving performance of dynamic programming via parallelism and locality on multicore architectures. IEEE Transactions on Parallel and Distributed Systems, 20(2):261-274, Feb. 2009. ISSN 1045-9219.

Y. Tan and K. Ding. A survey on GPU-based implementation of swarm intelligence algorithms. IEEE Transactions on Cybernetics, 46(9):2028-2041, 2016.

S. Taoka, D. Takafuji, and T. Watanabe. Enhancing PC cluster-based parallel branch-and-bound algorithms for the graph coloring problem. IEICE Transactions on Fundamentals of Electronics Communications and Computer Sciences, E91A(4):1140-1149, Apr. 2008. ISSN 0916-8508. doi: 10.1093/ietfec/e91-a.4.1140. 
D. Thiruvady, A. T. Ernst, and G. Singh. Parallel ant colony optimization for resource constrained job scheduling. Annals of Operations Research, 242(2):355-372, July 2016. ISSN 0254-5330. doi: 10.1007/s10479-014-1577-7.

U. Tosun, T. Dokeroglu, and A. Cosar. A robust island parallel genetic algorithm for the quadratic assignment problem. International Journal of Production Research, 51(14):4117-4133, July 2013. ISSN 0020-7543. doi: 10.1080/00207543.2012.746798.

M. Toulouse, T. G. Crainic, and K. Thulasiraman. Global optimization properties of parallel cooperative search algorithms: a simulation study. Parallel Computing, 26(1):91-112, 2000.

M. Toulouse, T. G. Crainic, and B. Sansó. Systemic behavior of cooperative search algorithms. Parallel Computing, 30 (1):57-79, 2004.

Q.-N. Tran. Designing efficient many-core parallel algorithms for all-pairs shortest-paths using CUDA. In Proceedings of the 7th International Conference on Information Technology: New Generations (ITNG), pages 7-12, 2010.

O. Trelles and A. Rodriguez. Bioinformatics and parallel metaheuristics, chapter 21, pages 517-549. John Wiley \& Sons, Inc., Hoboken, New Jersey, 2005. ISBN 9780471739388.

S. Tsutsui. Parallel ant colony optimization for the quadratic assignment problems with symmetric multi processing. In Proceedings of the 6th International Conference Ant Colony Optimization and Swarm Intelligence, pages 363-370. Springer-Verlag Berlin, 2008.

W. Tu, Q. Li, Q. Li, J. Zhu, B. Zhou, and B. Chen. A spatial parallel heuristic approach for solving very large-scale vehicle routing problems. Transactions in Gis, 21(6):1130-1147, Dec. 2017. ISSN 1361-1682. doi: 10.1111/tgis. 12272.

A. Umbarkar, M. S. Joshi, and W.-C. Hong. Multithreaded parallel dual population genetic algorithm (mpdpga) for unconstrained function optimizations on multi-core system. Applied Mathematics and Computation, 243:936-949, 2014.

E. Vallada and R. Ruiz. Cooperative metaheuristics for the permutation flowshop scheduling problem. European Journal of Operational Research, 193(2):365 - 376, 2009. ISSN 0377-2217. doi: https://doi.org/10.1016/j.ejor.2007.11.049. URL http://www.sciencedirect.com/science/article/pii/S0377221707011253

T. Van Luong, E. Taillard, N. Melab, and E.-G. Talbi. Parallelization strategies for hybrid metaheuristics using a single GPU and multi-core resources. In Proceedings of the 12th International Conference on Parallel Problem Solving from Nature, pages 368-377. Springer-Verlag Berlin, 2012.

T. Van Luong, N. Melab, and E.-G. Talbi. GPU computing for parallel local search metaheuristic algorithms. IEEE transactions on computers, 62(1):173-185, 2013.

P. Vidal, E. Alba, and F. Luna. Solving optimization problems using a hybrid systolic search on GPU plus CPU. Soft Computing, 21(12):3227-3245, 2017. ISSN 1432-7643.

T.-t. Vu and B. Derbel. Parallel branch-and-bound in multi-core multi-CPU multi-GPU heterogeneous environments. Future Generation Computer Systems-the International Journal of Escience, 56:95-109, Mar. 2016. ISSN 0167-739X. doi: 10.1016/j.future.2015.10.009.

C. Wang, D. Mu, F. Zhao, and J. W. Sutherland. A parallel simulated annealing method for the vehicle routing problem with simultaneous pickup-delivery and time windows. Computers \& Industrial Engineering, 83:111-122, May 2015. ISSN 0360-8352. doi: 10.1016/j.cie.2015.02.005.

D. Wang, C.-H. Wu, A. Ip, D. Wang, and Y. Yan. Parallel multi-population particle swarm optimization algorithm for the uncapacitated facility location problem using OpenMP. In Proceedings of the 2008 IEEE Congress on Evolutionary Computation, pages 1214-1218, 2008.

K. Wang, Z. Shen, et al. A GPU-based parallel genetic algorithm for generating daily activity plans. IEEE Trans. Intelligent Transportation Systems, 13(3):1474-1480, 2012.

M. Weber, F. Neri, and V. Tirronen. Shuffle or update parallel differential evolution for large-scale optimization. Soft Computing, 15(11):2089-2107, 2011.

K.-c. Wei, X. Sun, H. Chu, and C.-C. Wu. Reconstructing permutation table to improve the tabu search for the PFSP on GPU. Journal of Supercomputing, 73(11):4711-4738, Nov. 2017. ISSN 0920-8542. doi: 10.1007/ s11227-017-2041-7.

F. Xhafa and B. Duran. Parallel memetic algorithms for independent job scheduling in computational grids. In Recent advances in evolutionary computation for combinatorial optimization, pages 219-239. Springer-Verlag Berlin, 2008.

Y. Xu, T. K. Ralphs, L. Ladanyi, and M. J. Saltzman. Computational experience with a software framework for parallel integer programming. INFORMS Journal on Computing, 21(3):383-397, SUM 2009. ISSN 1091-9856. doi: 10.1287/ijoc.1090.0347. 
Q. Yang, L. Fang, and X. Duan. RMACO: a randomly matched parallel ant colony optimization. World Wide Web: Internet and Web Information Systems, 19(6):1009-1022, Nov. 2016. ISSN 1386-145X. doi: 10.1007/ s11280-015-0369-6.

M. Yazdani, M. Amiri, and M. Zandieh. Flexible job-shop scheduling with parallel variable neighborhood search algorithm. Expert Systems with Applications, 37(1):678-687, Jan. 2010. ISSN 0957-4174. doi: 10.1016/j.eswa.2009. 06.007.

Y.-S. You. Parallel ant system for traveling salesman problem on GPUs. In Proceedings of 11th. International Conference on Genetic and Evolutionary Computation, pages 1-2, 2009.

B. Yu, Z. Yang, X. Sun, B. Yao, Q. Zeng, and E. Jeppesen. Parallel genetic algorithm in bus route headway optimization. Applied Soft Computing, 11(8):5081-5091, Dec. 2011a. ISSN 1568-4946. doi: 10.1016/j.asoc.2011.05.051.

B. Yu, Z. Z. Yang, and J. X. Xie. A parallel improved ant colony optimization for multi-depot vehicle routing problem. Journal of the Operational Research Society, 62(1):183-188, Jan. 2011b. ISSN 0160-5682. doi: 10.1057/jors.2009.161.

W.-J. Yu, J.-Z. Li, W.-N. Chen, and J. Zhang. A parallel double-level multiobjective evolutionary algorithm for robust optimization. Applied Soft Computing, 59:258-275, Oct. 2017. ISSN 1568-4946. doi: 10.1016/j.asoc.2017.06.008.

R. A. O. Ze-Shu, Z. H. U. Wan-Ying, and K. ZHANG. Solving graph coloring problem using parallel discrete particle swarm optimization on CUDA. DEStech Transactions on Engineering and Technology Research, (amsm), 2017.

X.-Y. Zhang, J. Zhang, Y.-J. Gong, Z.-H. Zhan, W.-N. Chen, and Y. Li. Kuhnmunkres parallel genetic algorithm for the set cover problem and its application to large-scale wireless sensor networks. IEEE Transactions on Evolutionary Computation, 20(5):695-710, Oct. 2016. ISSN 1089-778X. doi: 10.1109/TEVC.2015.2511142.

Y. Zhang, S. Wang, and G. Ji. A comprehensive survey on particle swarm optimization algorithm and its applications. Mathematical Problems in Engineering, 2015, 2015.

J. Zhao, Q. Liu, W. Wang, Z. Wei, and P. Shi. A parallel immune algorithm for traveling salesman problem and its application on cold rolling scheduling. Information Sciences, 181(7):1212-1223, 2011.

Y. Zhou, F. He, N. Hou, and Y. Qiu. Parallel ant colony optimization on multi-core SIMD CPUs. Future Generation Computer Systems, 2017. ISSN 0167-739X. doi: https://doi.org/10.1016/j.future.2017.09.073. URL http://www sciencedirect.com/science/article/pii/S0167739X16304289.

W. Zhu and J. Curry. Parallel ant colony for nonlinear function optimization with graphics hardware acceleration. In Proceedings of the 2009 IEEE International Conference on Systems, Man and Cybernetics, pages 1803-1808, 2009.

\section{A Literature selection process}

When invited by the editorial board of European Journal of Operational Research in 2018, we were recommended to concentrate on the last decade of literature whenever possible. Following this recommendation is particularly reasonable for the body of literature on parallel optimization in OR because it accounts for a massive growth in computing performance in this period and resulting substantial advances of studies published regarding algorithmic parallelization, parallel software implementation and achieved computational results.

We conducted a title search in the most renowned OR journals. More specifically, we considered those 49 OR journals which are ranked "A+", "A", "B" or "C" in the German VHB-JOURQUAL 3 ranking of the German Academic Association for Business Research [German Academic Association for Business Research (VHB)]; a complete list of these journals is included in Table 5. As we expected to find research related to parallel optimization in OR also in journals that are dedicated to parallel computing, we included the following four journals in our search: Journal of Parallel and Distributed Computing, International Journal of Parallel Programming, Parallel Programming and Parallel Processing and Applied Mathematics. We used Web of Science to conduct a title search for both sets of journals, using the following search string: 
(parallel* OR distributed OR "'shared memory" OR MPI OR OpenMP OR CUDA OR GPU OR SMP) AND NOT "parallel machine" 


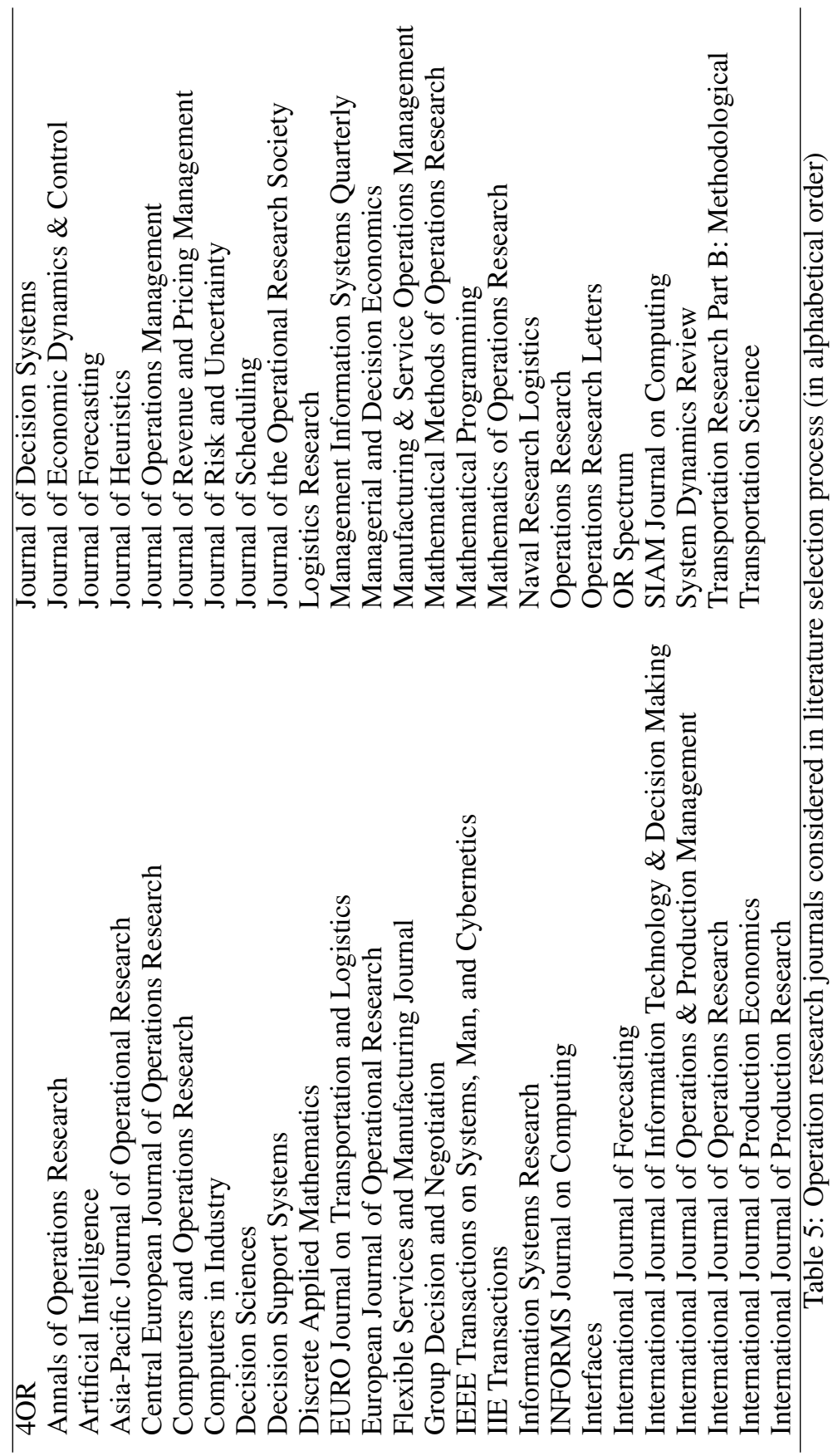


Acknowledging that research on parallel optimization relevant to the OR discipline is likely to be published also in journals of other disciplines and in conference proceedings and books, we also conducted a title search using Web of Science Core Collection without any restrictions regarding the publication outlet. However, we needed to adjust the search string in order keep the resulting list of articles manageable. The search strings that we used is as follows:

- "parallel* optimization" OR "parallel* branch" OR "parallel* discrete" OR "parallel heuristic" OR "parallel exact" OR "parallel meta" OR "parallel genetic" OR "parallel tabu" OR "parallel evolutionary" OR "parallel* ant colony" OR "parallel* simulated annealing" OR "parallel* variable neighborhood search" OR "parallel* Greedy Randomized Adaptive Search Procedures" OR "parallel* scatter search" OR "parallel* dynamic programming"

- (MPI OR OpenMP OR CUDA OR GPU) AND (heuristic* OR exact OR meta OR genetic OR branch OR optimization OR discrete OR tabu)

- (parallel* AND algorithm) AND (knapsack OR transport OR logistics OR evolutionary)

We also conducted a backward search of reference sections of literature reviews we identified (see the introduction of this article).

Overall, our literature search returned more than 1,100 entries. With the support of a $\mathrm{PhD}$ and several student workers, we used the title of an article to decide whether it should be excluded from further analysis due to a missing fit with the scope of this review, resulting in a preliminary list of 238 entries. Finally, with the help of the student workers we analyzed the content of each of these articles and excluded further 83 entries for a variety of reasons, including a missing fit with scope and the use of languages other than English. Finally, we conducted a backward search of reference sections of the remaining 155 articles to mitigate the risk of overlooking relevant studies: in a first step, we selected potentially relevant articles based on their title; in a second step, we analyzed the selected articles by inspecting the full text to decide whether they should be included in the final set of considered articles or not; this procedure yielded 50 additional articles. Overall, the ultimate set of articles, referred to as our sample, consists of 206 computational studies on parallel optimization in OR published between 2008 and 2017.

\section{B Coding of computational parallelization studies}

This section contains the detailed coding results of our sample with the exception of three studies: Östermark [2014. [2015] do not explicit the algorithm parallelized; Bozejko [2012] parallelizes the problem-specific evaluation of objective function but no overall algorithm is considered. To sum up, the tables in this section include 203 studies of the full sample (206 studies).

The articles are grouped along types of algorithms, with Table 6 addressing exact methods, Table 7 addressing single-solution based metaheuristics, Table 8 addressing population-based metaheuristics, Table 9 addressing hybrid metaheuristics, and Table 10 addressing problem-specific heuristics, other heuristics, and matheuristics. Unsurprisingly, not all studies included in our sample provide sufficiently precise details that allow coding all attributes. In cases where incomplete or ambiguous information is provided, we use the value "n/a". We need to point to two exceptions from this rule: 1) in the column "Process and search control", which show a triple classification, the usage of "n/a" for one or more of the three classes may confuse the reader. Thus, we prefer to use the symbol "?" where information 
is not available or ambiguous, or where our classification is not applicable (e.g., in reference [Derbel et al., 2014], a semi-synchronous mode is used because MPI-synchronization occurs at a pairwise level but not at a global level (p. 15)). 2) The entry "n/a" in the "Scalability" column has a more sophisticated interpretation, which we unfold in the text below.

The entries in the columns labeled "Problem" and "Algorithm" use the abbreviations as shown in Table 2 in the main text of the article. Entries in columns labeled "Parallelization strategy", "Process \& search control", "Communication topology" and "Programming model" are used as described in the main text.

The column "Scalability" covers both speedup and efficiency. It shows different types of entries: speedup that is qualified by its type of efficiency is provided in the form "sublinear $(n=2-16)$ ", for example, where the range of $n$ indicating the numbers of parallel processing units used. Speedup that varies between (sub)linear and superlinear depending on tested instances is described accordingly. Speedup achieved with GPGPUs is given as a single value or as an interval. We do not qualify speedup in this case as the number of parallel working units (usually GPGPU threads) needs to be interpreted different from that counting other parallel working units (CPU threads, processes) because they differ substantially from a technological perspective. Also, for the same reason, the determination of efficiency of parallelization should not be computed as the ratio of speedup and the number of parallel processing units. The entry " $\mathrm{n} / \mathrm{a}$ " in the "Scalability" column is an umbrella type and can have several different meanings described below. When more than one experiment has been conducted (e.g, applying different (versions of) algorithms, different (sets of) benchmark instances, and/or different programming models), speedup information is numbered.

Reasons for labeling scalability as " $\mathrm{n} / \mathrm{a}$ " turned out to be appropriate for manifold reasons:

- Times are compared with theoretical serial times.

- Speedup is related to other parallel executed algorithms or to parallel execution of the same algorithm (for example, because the execution on a single processing unit was practically infeasible due to time limitations); i.e., we report only speedups (weak or relative) related to serial executions of algorithms.

- The type of reference execution is unknown.

- No speedup values are reported or tedious work is necessary to determine them from data reported.

- Speedup values are provided in in supplementary material which is inaccessible.

- Speedup values only refer to parts of algorithms.

- Running times must not be compared as i) different (hardware) machines/computing environments are used, or ii) different levels of objective functions are achieved by reference execution(s) and execution of parallel algorithm.

- Parallelization is conducted in a virtual environment where no physical parallelization occurs. Then, execution times are hardly comparable as parallel execution times will often be larger than sequential times due to parallelization overhead.

We do not qualify speedup (as "linear", for example) in the case of GPGPU as programming model as the number of parallel working units (usually GPGPU threads) needs to be interpreted different from that counting other parallel working units (CPU threads, processes) because they differ substantially from a technological perspective. Also, for the 
same reason, the determination of efficiency of parallelization should not be computed as the ratio of speedup and the number of parallel processing units. 


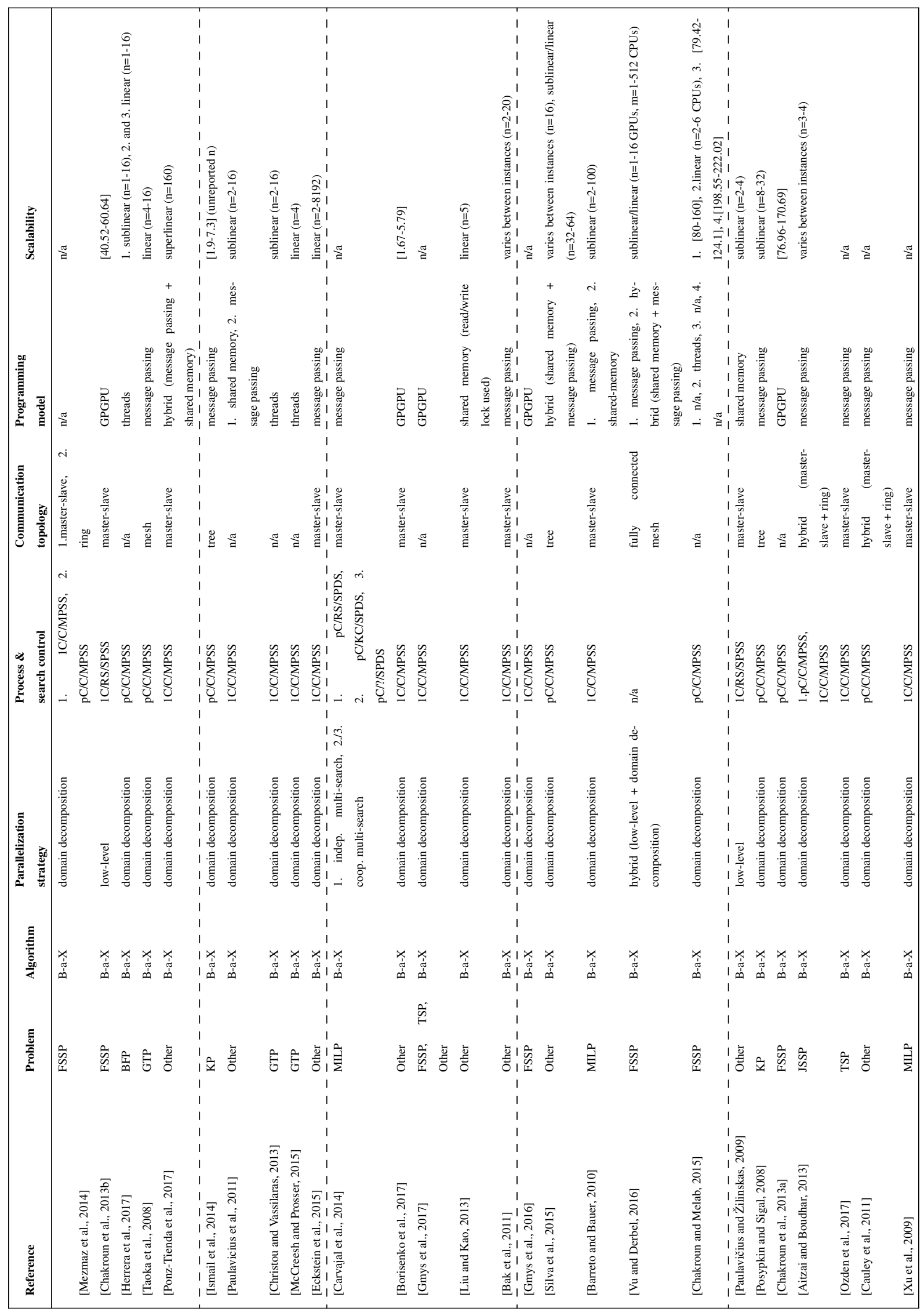




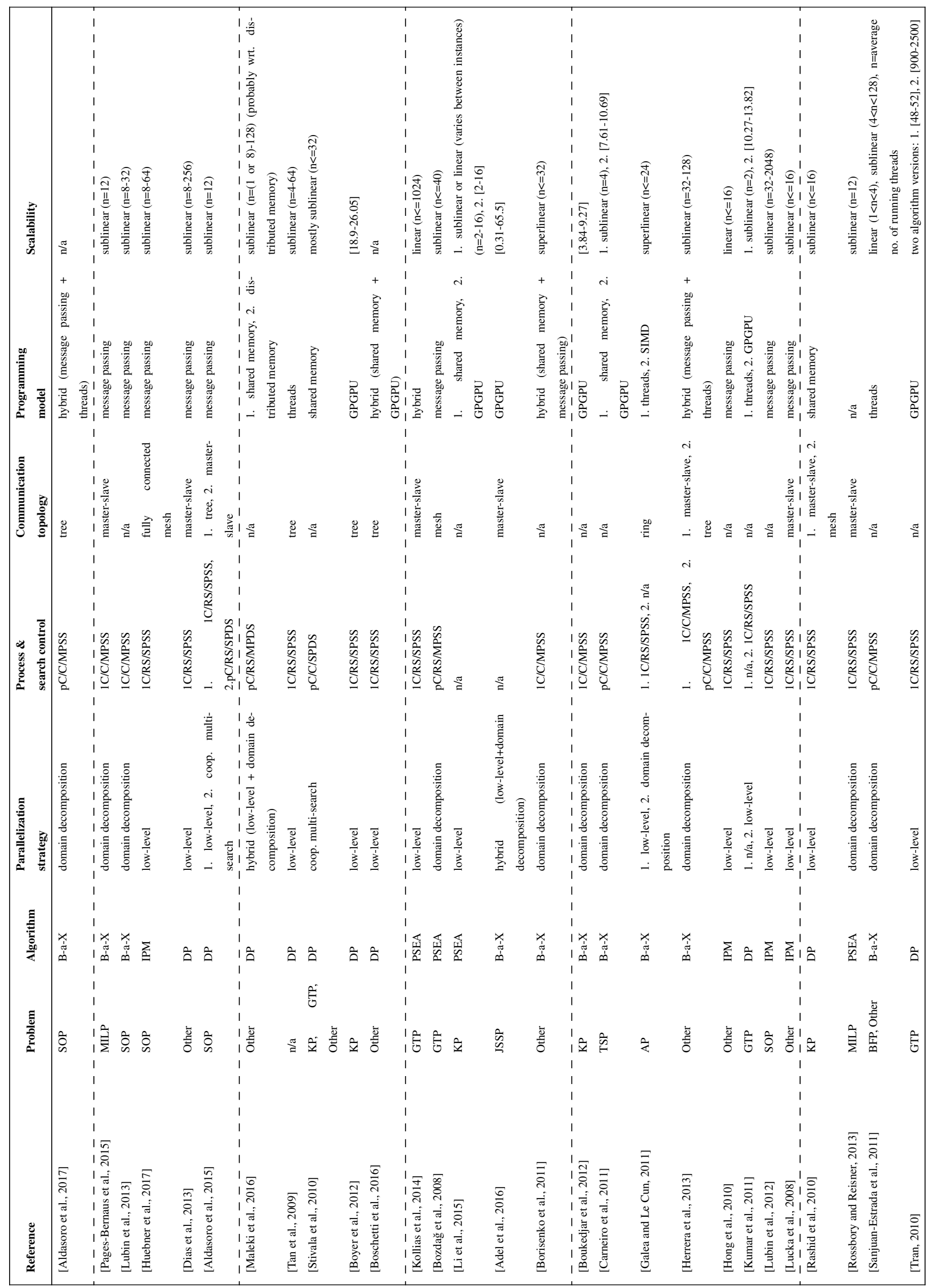




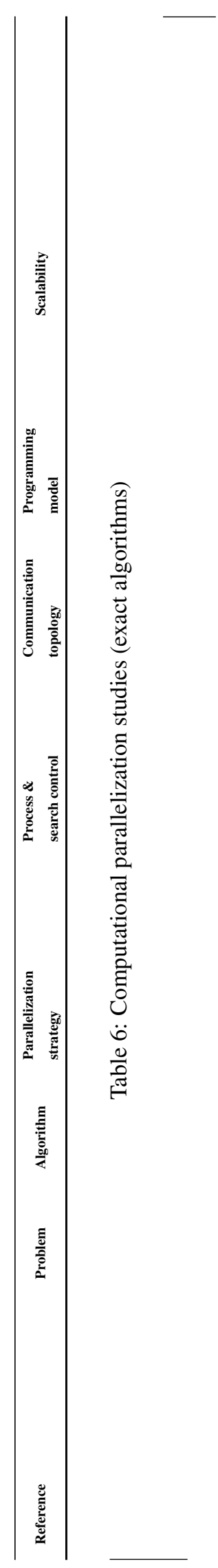




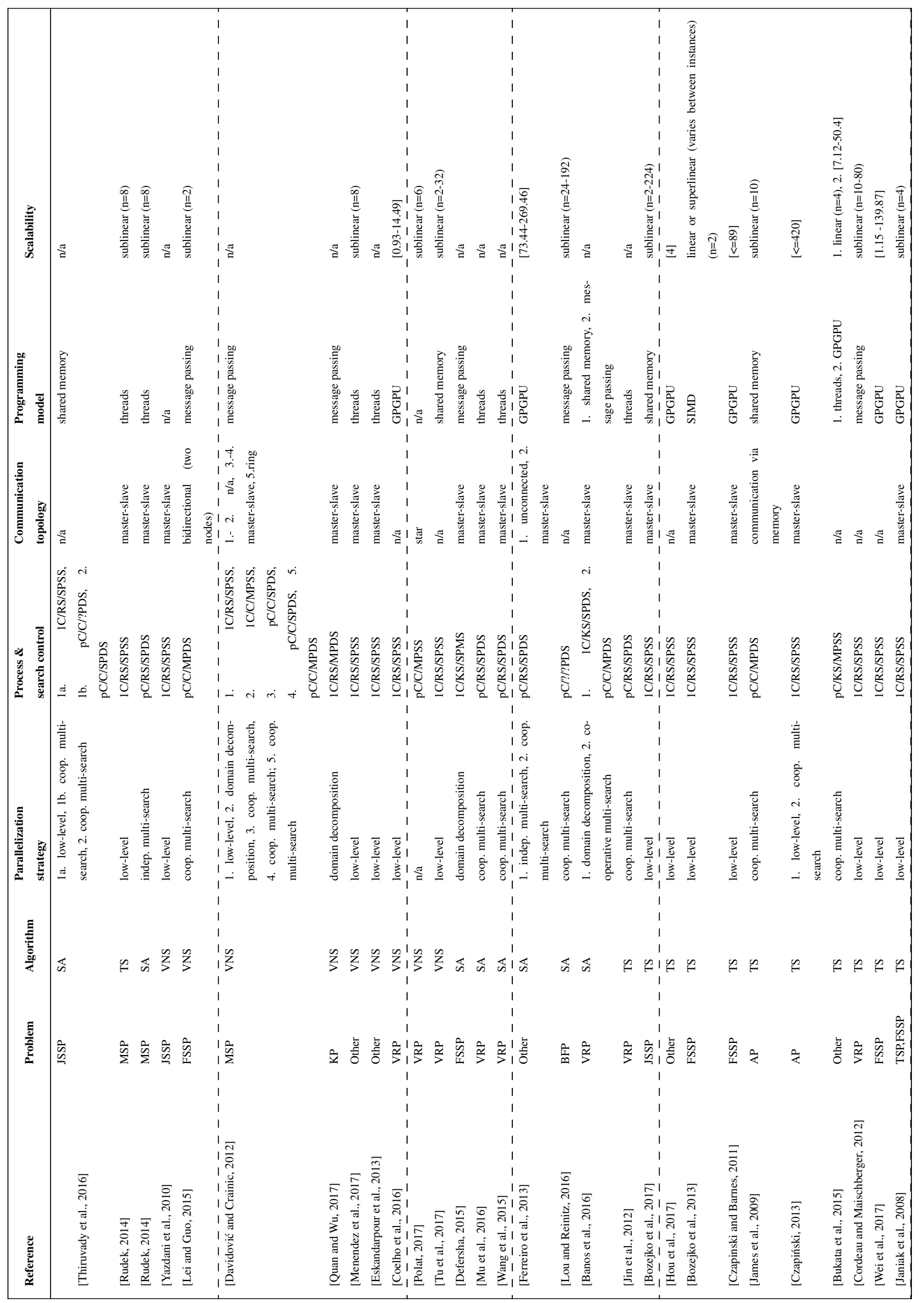




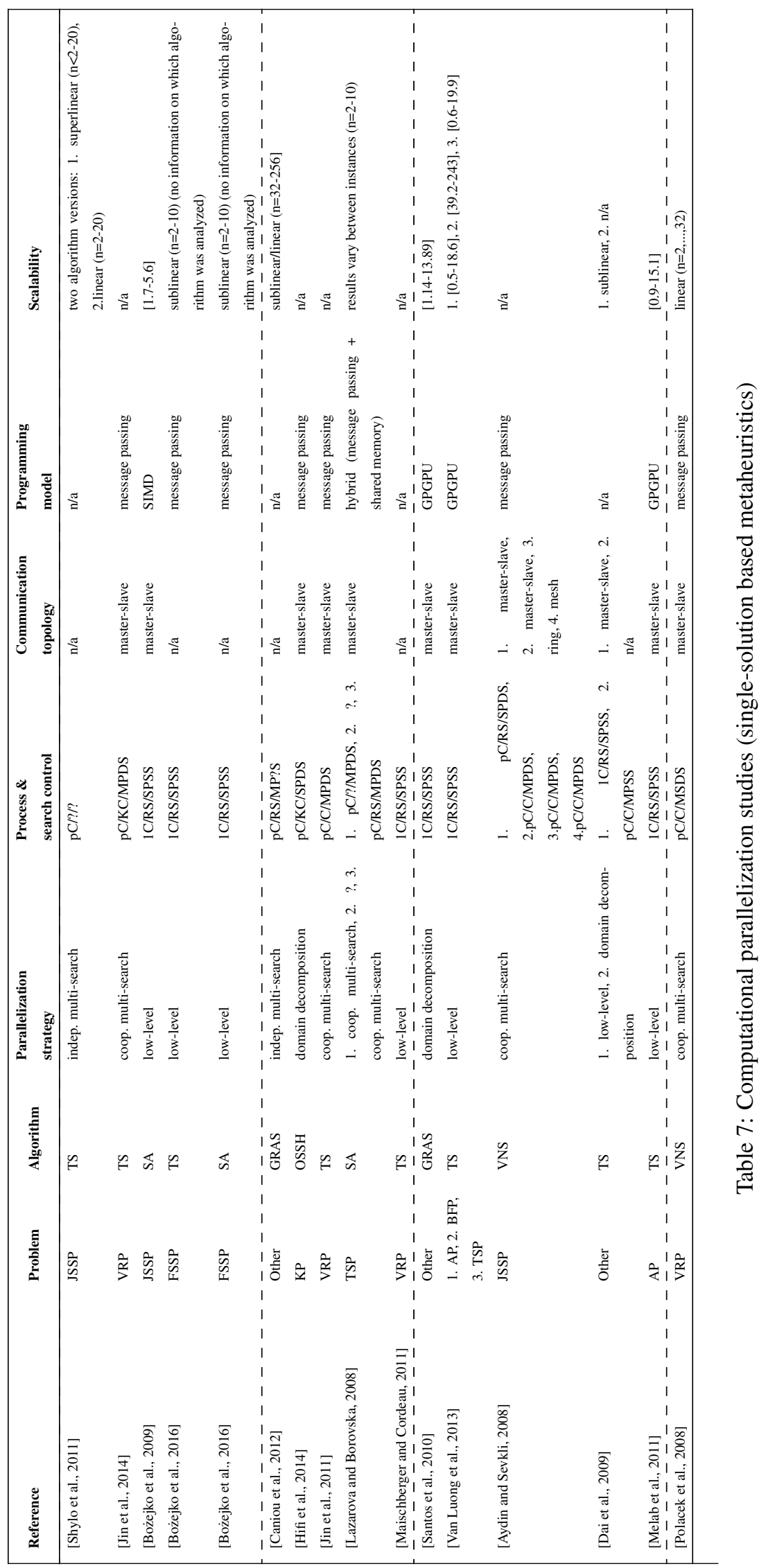




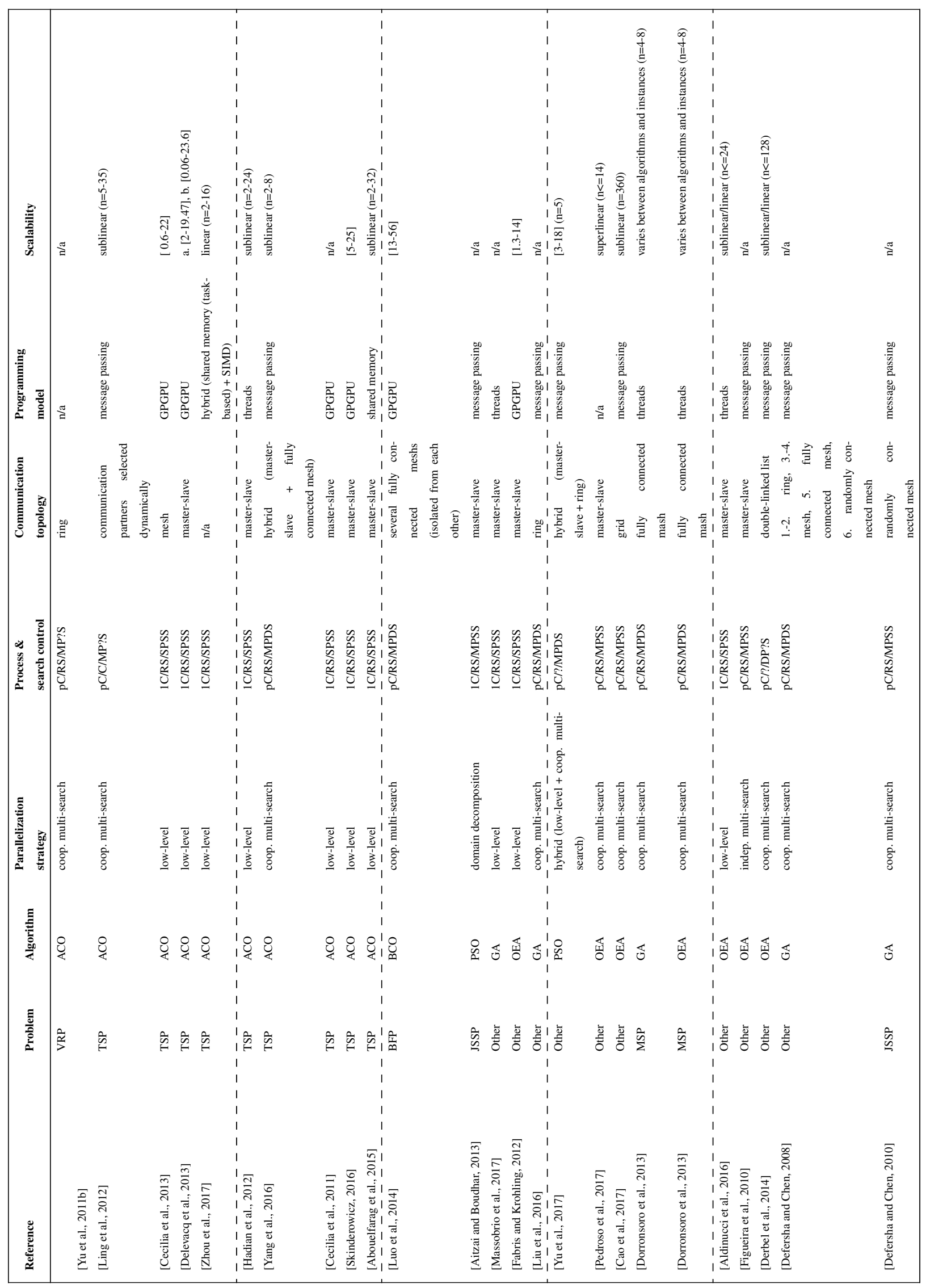




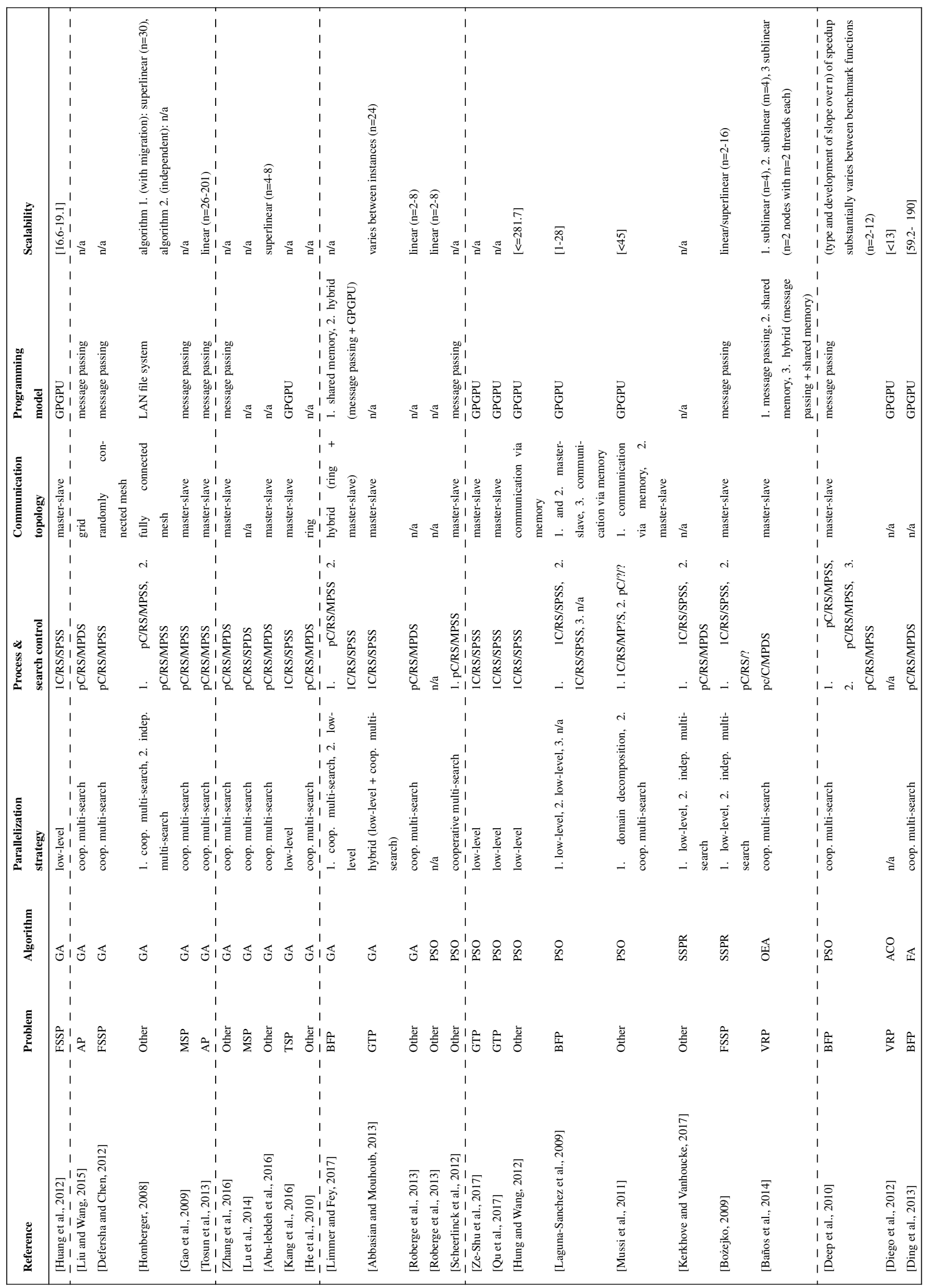




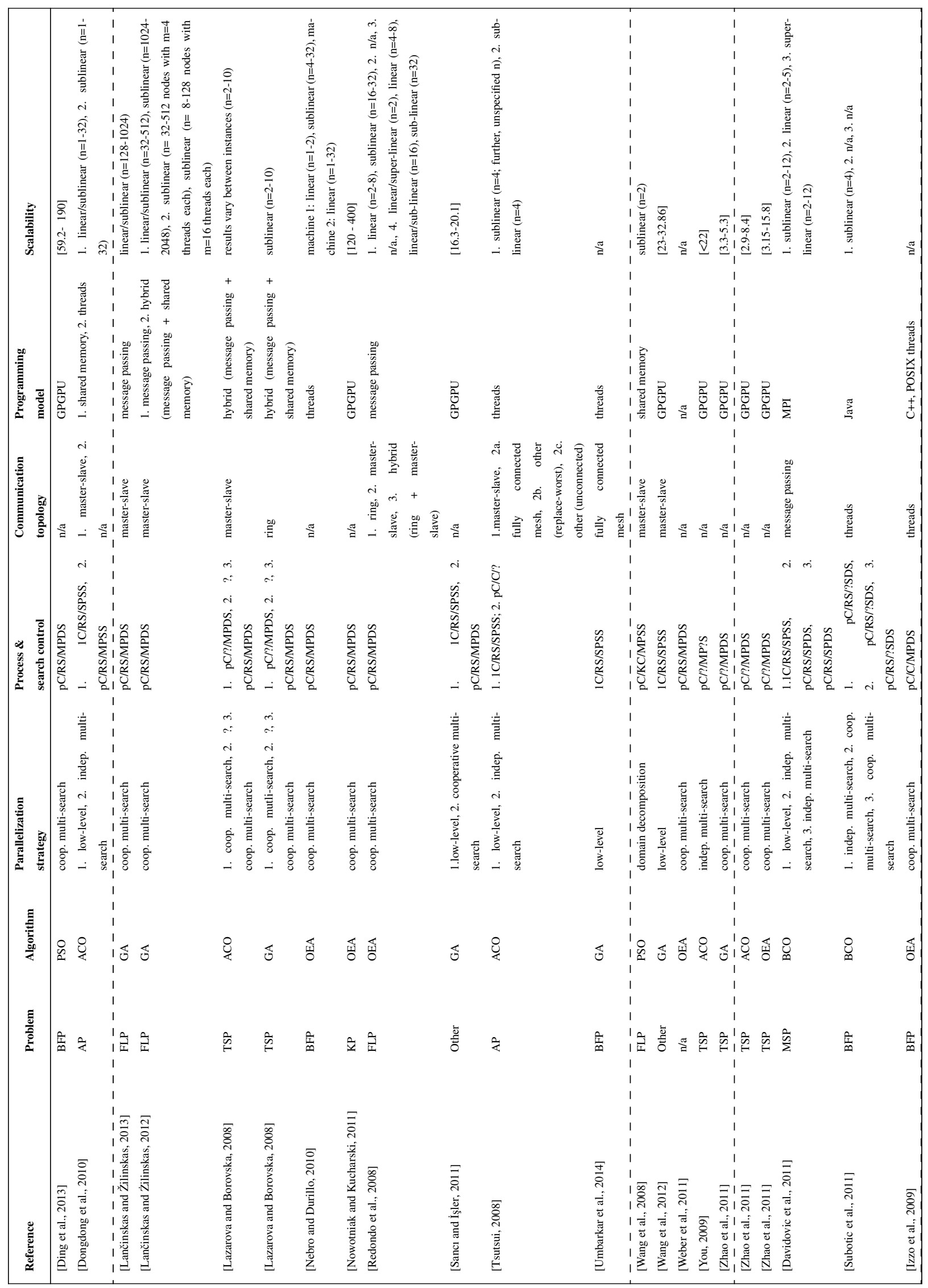




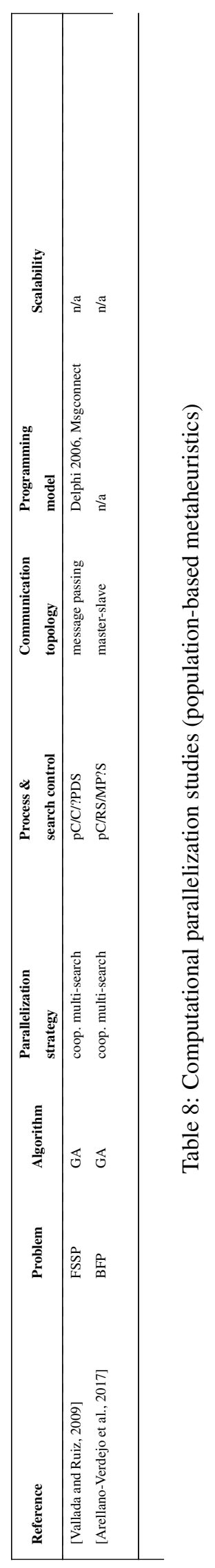




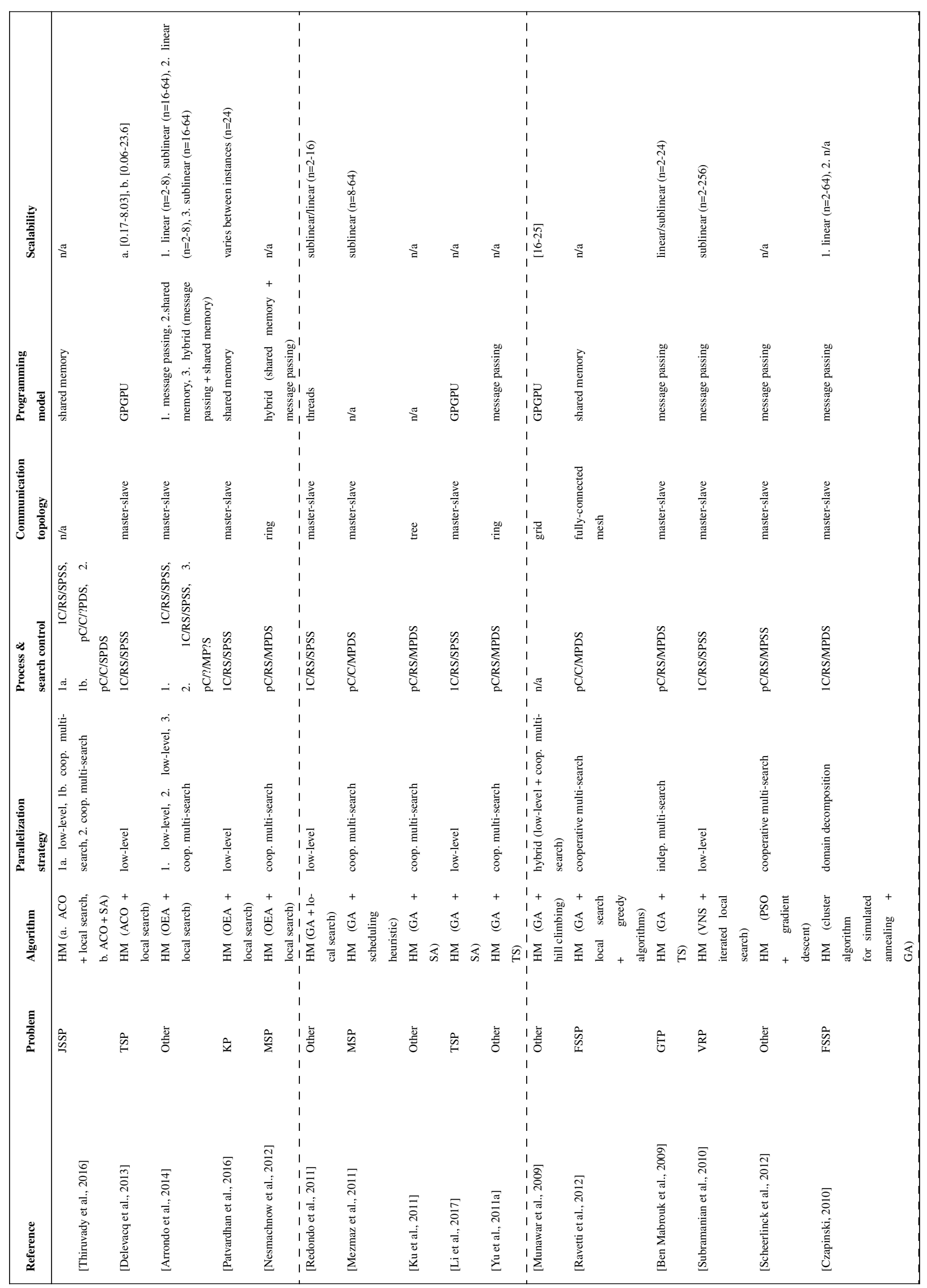




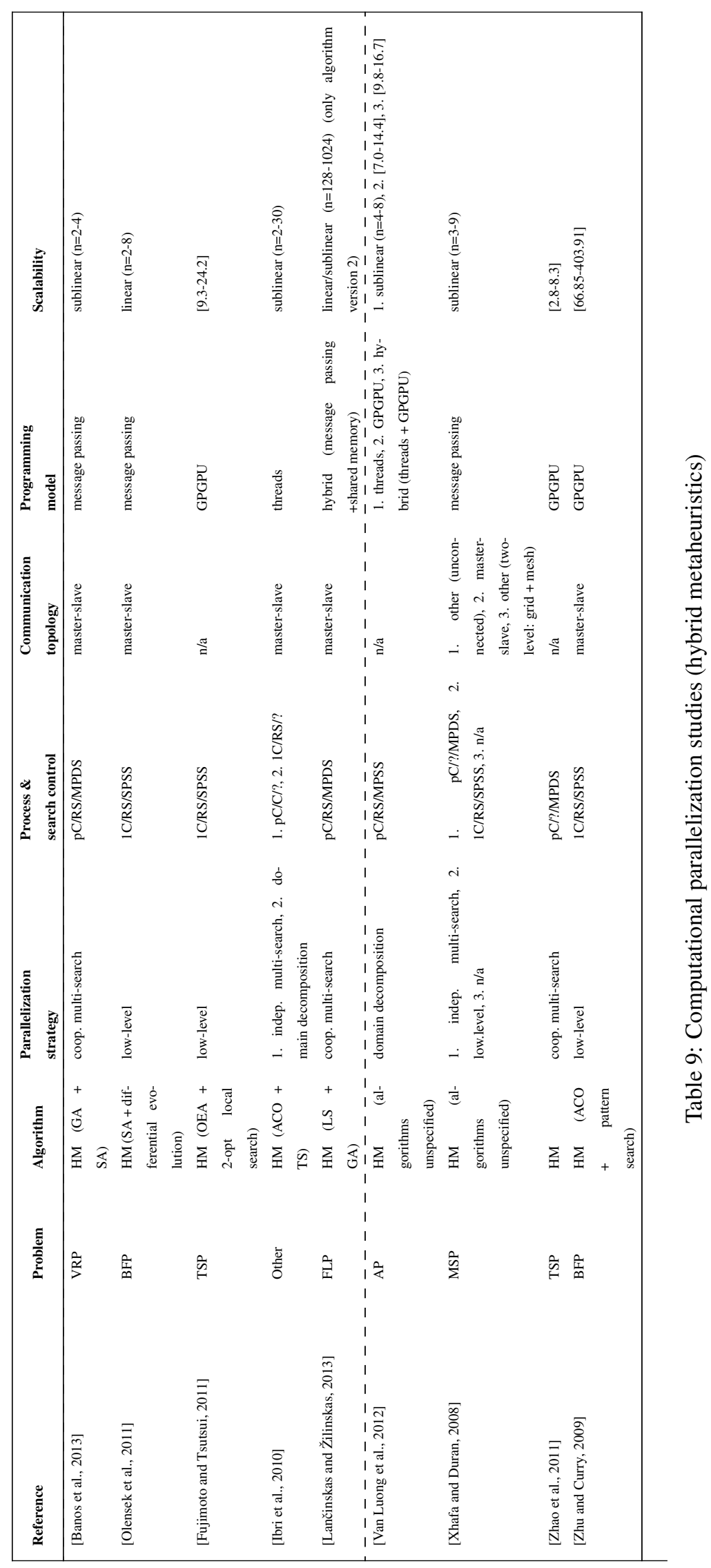




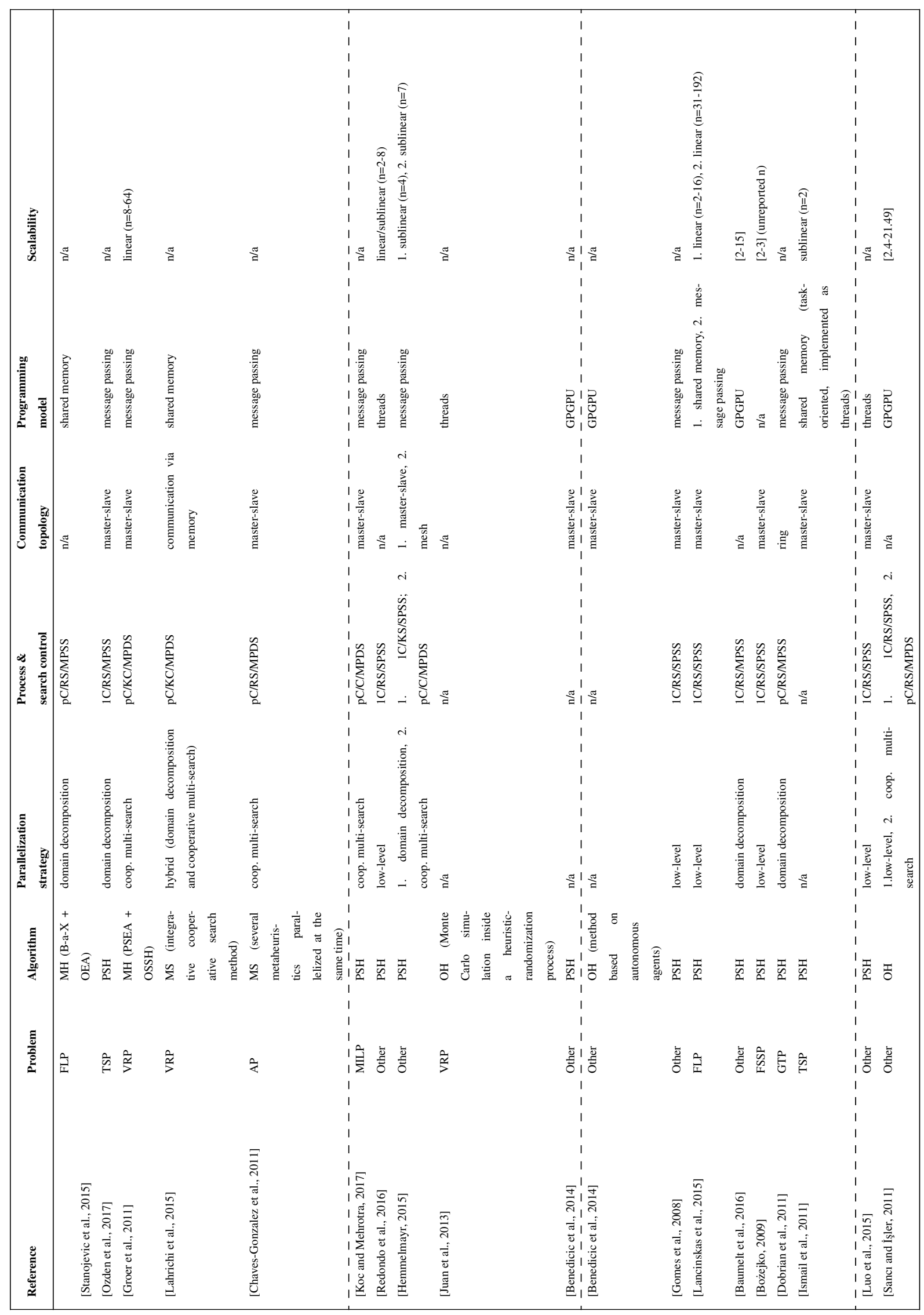




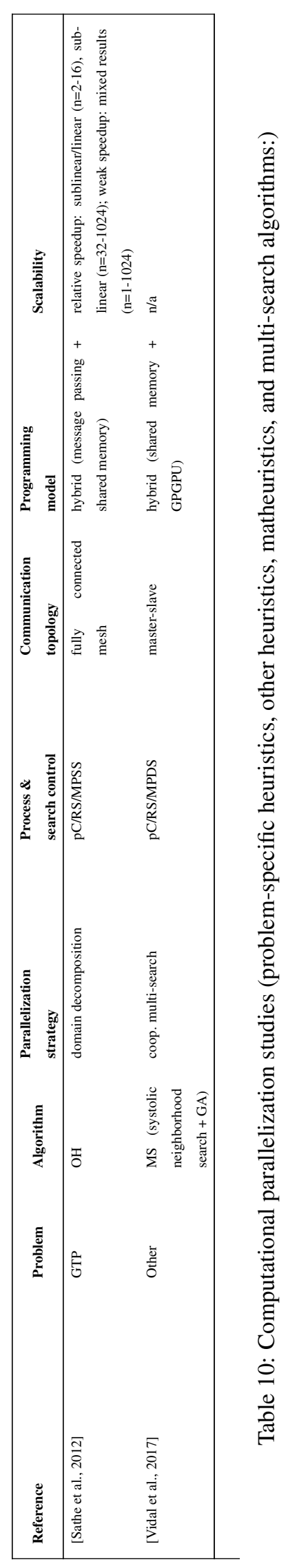




\section{References of Appendix}

Bozejko, W., 2012. On single-walk parallelization of the job shop problem solving algorithms. Computers \& Operations Research 39, 2258-2264.

German Academic Association for Business Research (VHB), . VHB-JOURQUAL3. http://vhbonline.org/vhb4you/jourqual/vhb-jourqual-3/teilrating-or/.

Östermark, R., 2014. Solving difficult mixed integer and disjunctive non-linear problems on single and parallel processors. Applied Soft Computing 24, 385-405.

Östermark, R., 2015. A parallel algorithm for optimizing the capital structure contingent on maximum value at risk. Kybernetes 44, 384-405. 